\title{
BOUNDEDNESS OF ONE-DIMENSIONAL BRANCHING MARKOV PROCESSES
}

\author{
F.I. KARPELEVICH ${ }^{1}$ \\ Moscow Institute of Transport Engineers (MIIT), The Russian Ministry \\ of Railways, 15 Obraztsova Str., Moscow 101475, Russia \\ YU. M. SUHOV ${ }^{2}$ \\ University of Cambridge, Statistical Laboratory \\ Department of Pure Mathematics and Mathematical Statistics \\ 16, Mill Lane \\ Cambridge CB2 1SB, UK \\ y.m.suhov@statslab.cam.ac.uk
}

(Received November, 1996; Revised May, 1997)

A general model of a branching Markov process on $\mathbb{R}$ is considered. Sufficient and necessary conditions are given for the random variable

$$
M=\sup _{t \geq 0} \max _{1 \leq k \leq N(t)} \Xi_{k}(t)
$$

to be finite. Here $\Xi_{k}(t)$ is the position of the $k$ th particle, and $N(t)$ is the size of the population at time $t$. For some classes of processes (smooth branching diffusions with Feller-type boundary points), this results in a criterion stated in terms of the linear ODE $\frac{\sigma^{2}(x)}{2} f^{\prime \prime}(x)+a(x) f^{\prime}(x)=$ $\lambda(x)(1-\kappa(x)) f(x)$. Here $\sigma(x)$ and $a(x)$ are the diffusion coefficient and the drift of the one-particle diffusion, respectively, and $\lambda(x)$ and $\kappa(x)$ the intensity of branching and the expected number of offspring at point $x$, respectively. Similarly, for branching jump Markov processes the conditions

\footnotetext{
${ }^{1}$ Supported by the American Mathematical Society and the International Science Foundation (Grant NM5E000) and by the INTAS Grant "Mathematical Methods for Stochastic Discrete Event Systems" (INTAS-93-820). Supported by St. John's College, Cambridge as a Michaelmas 1995 Overseas Visiting Scholar during OctoberDecember, 1995.

${ }^{2}$ Research supported in part by the EC Grant "Training Mobility and Research", No. 16296 (Contract CHRX-CT 93-0411) and the INTAS Grant "Mathematical Methods for Stochastic Discrete Event Systems" (INTAS-93-820), and carried out at the Institute for Mathematics and its Applications, University of Minnesota, Minneapolis, MN 55455, USA, and the Isaac Newton Institute for Mathematical Sciences, University of Cambridge, Cambridge CB3 0EH, UK.
} 
are expressed in terms of the relations between the integral $\mu(x) \int \pi(x, d y)(f(y)-f(x))$ and the product $\lambda(x)(1-\kappa(x)) f(x)$, where $\lambda(x)$ and $\kappa(x)$ are as before, $\mu(x)$ is the intensity of jumping at point $x$, and $\pi(x, d y)$ is the distribution of the jump from $x$ to $y$.

Key words: Branching Markov Process, Branching Diffusion, Branching Jump Markov Process, Boundedness, Green Measure, Green Operator, Generator.

AMS subject classifications: $60 \mathrm{~K} 35,60 \mathrm{~J} 80$.

\section{Introduction and Comments}

1.1 In this paper, the term branching Markov (BM) process is used for a continuous time Markov-type evolution of a system of randomly moving particles on the line $\mathbb{R}$. Particles may create new offspring; the rate of creation and the offspring number distribution depend only on the position of the parent particle. Each new particle starts moving from the point where it was created, in accordance with a fixed Markov process of individual motion (IM), which is not supposed to be space-homogeneous.

For the formal construction of a BM process and its basic properties we refer to Athreya and Ney [1]; some more recent works are discussed, e.g., in the review by Dynkin [6]. In particular, the reader is referred to Dynkin et al. [7], where general construction and properties of a measure-valued branching Markov process are discussed. Specific problems arising in the non-homogeneous and non-Euclidean cases are discussed in Lalley and Sellke [16] (see also the references therein) and Lalley and Sellke [17]. In this paper, we use the standard facts about the BD without going into detail. The IM Markov process is denoted by $\xi(=\xi(t), t \geq 0)$. The distribution of process $\xi$, given that it starts at point $x \in \mathbb{R}$, is denoted by $\mathbf{P}_{x}$, and the expectation in $\mathbf{P}_{x}$ is denoted by $\mathbf{E}_{x}$. The rate (intensity) of the branching of the particle positioned at $x$ is denoted by $\lambda(x)$, and the distribution of the number of offspring by $K(x, \cdot)$. We agree to think that a particle "dies" at the moment of branching, therefore it is not counted afterwards. By $\kappa(x)$ we denote the expected number of offspring produced at point $x: \kappa(x)=\sum_{j} K(x, j) j$. Throughout the paper we assume that $\lambda \in C^{0}(\mathbb{R})$
and

$$
0<\lambda_{0} \leq \lambda \leq \lambda_{1}<\infty, 1<\kappa_{0} \leq \kappa \leq \kappa_{1}<\infty,
$$

where $\lambda_{i}, \kappa_{i}$ are constants, $i=1,2$. [Here, and below, $C^{i}(\mathbb{R})$ denotes the space of functions on $\mathbb{R}$ which are of class $C^{i}$ at each point $x \in \mathbb{R}$ (without any assumption of boundedness); a similar notation is used when the line $\mathbb{R}$ is replaced by its subset.] We also assume that

$$
K(\cdot, 0)=0,
$$

which means that the particles always produce at least one offspring.

A number of interesting examples are incorporated if we assume that the IM process $\xi$ may have an "absorbing" state, $\star$, such that $\xi$ remains at $\star$ once it reaches it. We agree to think of $\star$ as a "point at $-\infty$ ", meaning formally that $\star<\infty$ and, moreover, $\star \in(-\infty, x)$ for any $x \in \mathbb{R}$. The "extended" line $\{\star\} \cup \mathbb{R}$ is denoted by $\overline{\mathbb{R}}$.

It is convenient to fix a phase space of the process (i.e., a subset $\mathbb{S} \subseteq \overline{\mathbb{R}}$ such that $\mathbf{P}_{x}\left(\xi_{t} \in \mathbb{S} \forall t>0\right)=1$ for each $\left.x \in \mathbb{S}\right)$. In the sequel, we work within $\mathbb{S}$ without mentioning this fact explicitly every time. It is assumed that $\mathbb{S} \cap \mathbb{R}$ is a Borel set 
which contains a sequence $z_{n}$ with $\lim _{n \rightarrow \infty} z_{n}=\infty$. It is also assumed that, for each $x \in \mathbb{S}, \quad \mathbf{P}_{x}$ is concentrated on the set $O_{x}$ of the right-continuous trajectories $\omega:[0, \infty) \rightarrow \mathbb{S}$ with $\omega(0)=x$. Finally, we suppose that $\xi$ is a Feller process on $\mathbb{S}$ (in the topology induced by the standard Euclid topology on $\mathbb{R})$; i.e., the corresponding semigroup $\left\{T_{t}: t \geq 0\right\}$ given by $T_{t} f(x)=\mathbf{E}_{x} f(\xi(t))$ takes space $C(\mathbb{S})$ of the bounded continuous functions $\mathbb{S} \mapsto \mathbb{R}$ to itself and is strongly continuous there. Thus, $\xi$ is determined by a generator $\mathfrak{G}$ which is a densely defined operator $\operatorname{in} C(\mathbb{S})$. While dealing with some particular types of processes (a smooth diffusion on $\mathbb{R}$ or on $\overline{\mathbb{R}}_{+}=$ $\{\star\} \cup[0, \infty)$ with a boundary point at 0$)$, it is convenient to treat $\mathfrak{G}$ as an operator in the space $D\left(=D_{\xi}(\mathbb{R})\right.$ or $\left.D_{\xi}(\mathbb{R}+)\right)$. See Section 3 below.

The BM process is denoted by $\Xi(t)$, and its sample space and probability distribution, given that the starting point is $x$, by $\Omega_{x}$ and $\mathscr{P}_{x}$, respectively. Given $t \geq 0$, let $\Xi_{k}(t), k=1, \ldots, N(t)$, denote the random positions of particles by time $t$, where $N(t)$ is the total number of particles in the population. We are interested in the quantity

where

$$
M=\sup _{t \geq 0} Y(t)=\sup _{t \geq 0} Y^{(t)}
$$

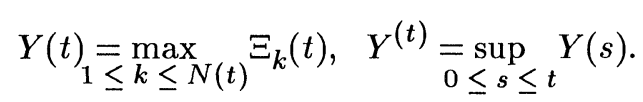

Here, the random variable $Y(t)\left(Y^{(t)}\right)$ gives the maximal position of the particles in the BM process at time $t$ (respectively, on the time interval $[0, t])$ and $M$ the overall supremum of the positions. The question is whether the random variable $M$ is proper, i.e.,

$$
\mathscr{P}_{y}(M<\infty)=1, y \in \mathbb{R}
$$

A BM is called bounded (in a positive direction) if the variable $M$ is proper.

A similar question arises about the quantity

$$
\tilde{M}=\sup _{n \geq 0} \tilde{Y}(n)=\sup _{n \geq 0} \tilde{Y}^{(n)}
$$

where $\tilde{Y}(n)\left(\tilde{Y}^{(n)}\right)$ gives the maximum of the position of the particles of the $n$th generation (respectively, the generations $0,1, \ldots, n$ ) in the BM process, at the times of their divisions $T_{1}, \ldots, T_{\widetilde{N}(n)} ; \widetilde{N}(n)$ is the number of particles of the $n$th generation. [The $n$th generation is formed by particles that have precisely $n$ ancestors.] By definition, $\widetilde{Y}(0)=\widetilde{Y}^{(0)}=x$. We say that a BM process is quasibounded (in a positive direction) if the random variable $\tilde{M}$ is proper.

It is clear that if a BM process is bounded, it is quasibounded. We show that if the IM process $\xi$ is a smooth diffusion (possibly with a boundary point), the converse is also true.

Intuitively, the answer to both questions depends on the outcome of a "competition" between several factors: the "drift" and "volatility" of the law of process $\xi$ and the parameters $\lambda$ and $K$ of the branching mechanism. In Section 2 we give general sufficient and necessary conditions for boundedness and quasiboundedness of a BM process under quite general assumptions on $\xi$. These conditions are stated in the form of inequalities involving the so-called Green measures of process $\xi$ and its stopped version. [The conditions on $\xi$ imposed in the necessity part are more restrictive than in the sufficiency part.]

An interesting example of the BM process is the branching diffusion (BD), where $\xi$ is a diffusion process. We consider the case where $\xi$ is a "smooth" diffusion on 
$\mathbb{S}=\mathbb{R}$ or $\overline{\mathbb{R}}_{+}$, where $\overline{\mathbb{R}}_{+}=\{\star\} \cup \mathbb{R}{ }_{+}$and $\mathbb{R}_{+}=[0, \infty)$. In the latter case we assume that 0 is a Feller-type boundary point. In Section 3 we establish a criterion (i.e., a necessary and sufficient condition) which is quite unexpectedly short: let $\mathfrak{G}_{\text {lo }}$ denote the "local" generator of process $\xi$, with a domain $D\left(\mathfrak{G}_{\mathrm{lo}}\right)$. "Local" means that no assumption is made about the behavior of functions $f \in D\left(\mathfrak{S}_{\text {lo }}\right)$ as $x \rightarrow \pm \infty$; all conditions are stated in terms of their properties in some neighborhoods of the points $x \in \mathbb{S}$. In other words, $\mathfrak{G}_{\mathrm{lo}}$ is merely a second order differential operator, with the diffusion coefficient $\sigma^{2}(x)$ and drift $a(x)$, completed, when necessary, with the corresponding boundary condition. Formally, $\mathfrak{G}_{\text {lo }}$ is an extension of the "global" generator 5. [The precise meaning is given in Section 3.] Then there is the following necessary and sufficient condition, called below Condition (or Criterion) CD for the $\mathrm{BD}$ process to be bounded (and hence, quasibounded).

There exists a function $f^{0}$ (on $\mathbb{R}$ or $\overline{\mathbb{R}}_{+}$) such that

and

$$
f^{0} \geq 0, \lim _{x \rightarrow \infty} f^{0}(x)=\infty,
$$

$$
f^{0} \in D\left(\mathfrak{G}_{\mathrm{lo}}\right), \mathfrak{S}_{\mathrm{lo}} f^{0}(x)+\lambda(x)(\kappa(x)-1) f^{0}(x)=0 .
$$

Thus, Criterion CD reduces a rather intricate probability-type question about a BD process to a problem concerning a spectral property of a linear differential operator. In Sections 1.2-1.5 we comment on the applicability of Criterion CD, and show that in a number of interesting examples it leads to a straightforward answer.

The problem of boundedness (or, more generally, of an asymptotical behavior) of a BM process was first discussed, in a somewhat different setting, by Hammersley [9] and Kingman [15]; later it was actively investigated by Biggins and co-authors (see $[2,3]$ and the references therein). However, the above papers considered the case of a space-homogeneous IM process $\xi$, constant $\lambda$, and fixed probability distribution $K$. A general model of a discrete-time and one-dimensional discrete-space non-homogeneous BM process was recently discussed by Karpelevich and Suhov (see [14] and the references therein), where a "discrete" analog of Condition CD was derived.

1.2 We start our cornments on Criterion CD with the case of a space-homogeneous $\mathrm{BD}$ process, where $\xi$ has a constant drift and diffusion coefficients $a \in \mathbb{R}$ and $\sigma>0$, and $\lambda$ and $K$ (and hence $\kappa$ ) do not depend on $x \in \mathbb{R}$. In this case, we speak of a branching Brownian (BB) motion. Then, if $\kappa>1$, a necessary and sufficient condition of boundedness is:

$$
a<0, \frac{a^{2}}{\sigma^{2}} \geq 2 \lambda(\kappa-1) .
$$

Bound (1.8) follows from results of McKean [18]. See also Karpelevich et al. [12]. [Earlier, it was established in Biggins [2] that under (1.8), the space-homogeneous BB motion is quasibounded; the fact that (1.8) is necessary for quasiboundedness also may be derived from results of [2].]

It is easy to check that (1.8) is equivalent to Condition CD. [Of course, the BB motion will then be unbounded in a negative direction.] In fact, in this case $\mathfrak{F}_{\text {lo }}$ is a second-order differential operator on $\mathbb{R}$ with constant coefficients

$$
\mathfrak{G}_{\mathrm{lo}} f(x)=\frac{\sigma^{2}}{2} f^{\prime \prime}(x)+a f^{\prime}(x), x \in \mathbb{R} .
$$


The general solution to the linear $\operatorname{ODE}(1.7)$ is of the form $c_{+} \exp \left(b_{+} x\right)+$ $c_{-} \exp \left(b_{-} x\right), x \in \mathbb{R}$, where $c_{ \pm}$are constants, and $b_{ \pm}=-\frac{a}{\sigma^{2}} \pm\left(\frac{a^{2}}{\sigma^{4}}-\frac{2 \lambda(\kappa-1)}{\sigma^{2}}\right)^{1 / 2}$, which immediately leads to (1.8). Note that under (1.8), $b_{+} \geq b_{-}>0$.

Pictorially speaking, the ratio $a^{2} / \sigma^{2}$ in (1.8) measures the "rigidity" of a BM: the greater it is, the less "random" the motion looks at large times. The value $\frac{a^{2}}{\sigma^{2}}-2 \lambda(\kappa-1)$ shows the outcome of the "competition" between the rigidity and an "entropy" of the process of creating new particles. In short, the meaning of condition (1.8) is that the drift suppresses all "entropy factors" presented in the BB motion.

Criterion CD provides a straightforward answer to the question of boundedness in all cases where the operator $\mathfrak{S}_{\text {lo }}$ may be written in the form (1.9) after a change of variables. In other words, it means that what matters for boundedness of a $\mathrm{BM}$ process is the behavior of certain expectation values as functions of a space variable. This covers a variety of examples where, to our knowledge, none of the existing methods is applicable.

1.3 Another example where the Criterion CD gives a complete answer while other existing methods do not is the $\mathrm{BB}$ motion on $\overline{\mathbb{R}}_{+}$. Suppose again that $a, \sigma, \lambda$ and $\kappa$ are constant, $\sigma>0$, and consider the case of a Feller boundary point at 0 . The latter means that the boundary condition at point 0 for operators $\mathfrak{S}$ and $\mathfrak{S}_{\text {lo }}$ reads

$$
p_{1} f(0)-p_{2} f^{\prime}(0)+p_{3}\left(\frac{\sigma^{2}}{2} f^{\prime \prime}(0)+a f^{\prime}(0)\right)=0 .
$$

Here $p_{i} \geq 0, i=1,2,3$, are constants; $p_{1}+p_{2}+p_{3}=1$. The case $p_{1}=p_{3}=0$ corresponds to a reflecting and $p_{2}=p_{3}=0$ an absorbing (or killing) barrier at 0 ; in the latter case, process $\xi$ jumps from 0 to state $\star$ and remains there forever. The operator $\mathfrak{F}_{\mathrm{lo}}$ is given by the right-hand side of (1.9), by replacing $x \in \mathbb{R}$ by $x \geq 0$.

As before, the general solution to (1.7) (in the case under consideration it means that $f^{0}$ satisfies the differential equation and the boundary conditions) has, for $x \geq 0$, the above form. A simple analysis shows that if condition (1.8) is violated, the BB motion on $\mathbb{R}_{+}$is unbounded for all initial points $y>0$ and any choice of the boundary condition of the form (1.10). In other words, if the homogeneous version of the $\mathrm{BB}$ motion on the whole line is unbounded, then its half-line modification is also unbounded. On the other hand, if (1.8) holds (i.e., the BB motion on $\mathbb{R}$ is bounded), then its half-line version may be either bounded or unbounded: it depends on the choice of constants $p_{i}$ in (1.10). More precisely, under condition (1.8), the motion is bounded iff

$$
p_{1} \geq p_{2} b_{-}+p_{3} \lambda(\kappa-1) .
$$

Pictorially speaking, the coefficient $p_{1}$ "helps" to maintain the BB motion on $\overline{\mathbb{R}}_{+}$bounded, while $p_{2}, p_{3}$ "act" in an opposite direction. In particular, in the case of an absorbing barrier, the BB motion is bounded iff its whole-line version is bounded; in the case of a reflecting barrier, it is always unbounded. Observe that this statement is not true in the case where $K(\cdot, 1)=1$ (and hence $\kappa=1$ ). Here, the BB motion $\Xi$ is reduced to the IM process $\xi$. But $\xi$ on the half-line, with an absorbing barrier, is bounded for $a=0$, whereas $\xi$ on the whole line is not. 
1.4 Feller [8] considered an example of a Markov process $\xi$ on $\overline{\mathbb{R}}+$, where a particle moves as in a diffusion process on $\mathbb{R}_{+}$, with a boundary point at 0 , and in addition may jump from 0 to $(0, \infty)$. See also [11], Chapter 5, Section 5.7. The corresponding BM process $\Xi$ is called a branching diffusion with a jump (BDJ).

Criterion CD (where $\mathfrak{G}_{\text {lo }}$ is again understood as a second-order differential operator on $\mathbb{R}_{+}$, with specific boundary conditions) is also applicable for the BDJ processes (see Section 3). As before, it gives a particularly simple answer in the case where the diffusion on $\mathbb{R}_{+}$has constant coefficients, and $\lambda$ and $\kappa$ do not depend on $x \in \mathbb{R}_{+}$. More precisely, let $a$ be the drift and $\sigma$ the diffusion coefficients, and the boundary condition for the diffusion on $\overline{\mathbb{R}}_{+}$be of the form

$$
p_{1} f(0)-p_{2} f^{\prime}(0)+p_{3}\left(\frac{\sigma^{2}}{2} f^{\prime \prime}(0)+a f^{\prime}(0)\right)=\mu \int_{(0, \infty)} p(d y)(f(y)-f(0)) \text {. }
$$

Here $p_{i} \geq 0, i=1,2,3$, and $\mu \geq 0$ are constants, $p_{1}+p_{2}+p_{3}+\mu=1$, and $p$ is a probability distribution on $(0, \infty)$. Then the BDJ process $\Xi$ is bounded iff $(1.8)$ holds and

$$
p_{1} \geq p_{2} b_{-}+p_{3} \lambda(\kappa-1)+\mu \int_{(0, \infty)} p(d y)\left(\exp \left(b_{-} y\right)-1\right) .
$$

1.5 An interesting class is formed by the branching jump Markov (BJM) processes. Here, the IM process $\xi$ is a jump Markov process characterized by the jump intensity $\mu(x)$ and the distribution of the jump $\pi(x, d y), x, y \in \mathbb{S}$. In Section 4 we establish that the condition

There exists a function $f^{0}($ on $\mathbb{S})$ such that (1.6) holds and

$$
f^{\prime} \in D\left(\mathfrak{S}_{\text {in }}\right), \mathfrak{G}_{\text {in }} f^{0}(x)+\lambda(x)(\kappa(x)-1) f^{0}(x)=0
$$

is sufficient for the BJM to be bounded. Here, $\mathfrak{S}_{\text {in }}$ is an extension of the "global" generator $\mathfrak{F}$ (which is now an integral operator) to its maximal natural domain (see Section 4).

On the other hand, under some additional assumptions, the condition

There exists a function $f^{0}($ on $\mathbb{S})$ such that (1.6) holds and

$$
f^{0} \in D\left(\mathfrak{F}_{\text {in }}\right), \mathfrak{F}_{\text {in }} f^{0}(x)+\lambda(x)(\kappa(x)-1) f^{0}(x) \leq 0
$$

is necessary for quasiboundedness. The sufficient condition is called SJ and the necessary one NJ. The question of finding a general class of BJM processes for which Condition SJ is necessary for quasiboundedness or boundedness remains open.

Note that in the case of a discrete time/space BJM process (called a branching random walk), a criterion (i.e., a necessary and sufficient condition) of boundedness was established in Karpelevich et al. [13, 14]. The space-homogeneous case was investigated earlier in Karpelevich et al. [12].

1.7 The methods used in this work originate mainly in functional analysis (more precisely, semi-group theory) and the theory of ordinary second-order differential equations. [We refer the reader to Dynkin [5], Itô and McKean [11]; and Yosida [20] for the results from semi-group theory, and to Birkhoff and Rota [4], Hartman [10] and Taira [19] for the results from the theory of second-order ODE's and their connections with the theory of diffusion processes.] Probabilistic technique plays a rather subordinated role in the present paper. Nevertheless, we believe that an adequate 
approach based on probabilistic ideas is possible (and hope it will follow soon). For example, a process $\gamma(t)$ given by

$$
\gamma(t)=\sum_{k=1}^{N(t)} f^{0}\left(\Xi_{k}(t)\right)
$$

is a martingale iff function $f^{0}$ satisfies (1.7).

\section{Abstract Conditions of the Boundedness of a BM Process}

2.1 In Sections 2.1 and 2.2 we establish inequalities that give sufficient and necessary conditions of boundedness and quasiboundedness of a BM process on $\overline{\mathbb{R}}$. The proofs are given in Section 2.3. Sufficient inequalities are established in a fairly general situation and do not require specific assumption about process $\xi$. However, we assume that $\lambda$ and $K$ satisfy (1.1), (1.2).

We introduce the random variable $\rho\left(=\rho_{x}\right)$, on probability spaces $\left(\Omega_{x}, \mathbf{P}_{x}\right)$, describing the lifetime of the process started at $x$, when the rate of lifetime spending at point $y$ is $\lambda(y)$. Our analysis is focused on stochastic equations that are naturally connected with the variables $M$ and $\widetilde{M}$. More precisely, let $M_{x}$ and $\widetilde{M}_{x}$ denote the "versions" of these variables on $\left(\Omega_{x}, \mathscr{P}_{x}\right)$. Then

$$
M_{x} \sim \max \left[\eta_{x}, M_{\xi_{x}\left(\rho_{x}\right)}^{(1)}, \ldots, M_{\xi_{x}\left(\rho_{x}\right)}^{\left(j_{x}\right)}\right]
$$

and

$$
\tilde{M}_{x} \sim \max \left[x, \tilde{M}_{\xi_{x}\left(\rho_{x}\right)}^{(1)}, \ldots, \tilde{M}_{\xi_{x}\left(\rho_{x}\right)}^{\left(j_{x}\right)}\right]
$$

Here, symbol $\sim$ means equality in distribution. The random variable $\eta_{x}$ in the RHS of $(2.1 a)$ gives the value $\sup _{0 \leq t \leq \rho_{x}} \xi_{x}(t)$, and $j_{x}$ in the RHS of $(2.1 a, b)$ is the random number of offspring produced in a single act of division (at random pint $\xi_{x}\left(\rho_{x}\right)$ ). Furthermore, given that $\rho_{x}=y$ and $j_{x}=j$, the random variables $M_{\xi\left(\rho_{x}\right)}^{(i)}$ and $\tilde{M}_{\xi\left(\rho_{x}\right)}^{(i)}, i=1, \ldots, j$, are (conditionally) independent and have the same distribution as $M_{y}$ and $\tilde{M}_{y}$, respectively. It is easy to check that variables $M_{x}$ and $\tilde{M}_{x}, x \in \mathbb{S}$, give minimal solutions to $(2.1 a, b)$, in the sense of stochastic ordering.

Given $z \in \mathbb{R}$, let $\tau^{z}$ be the time when process $\xi$ hits $[z, \infty): \tau^{z}=\min [\widehat{t} \geq 0$ : $\xi(\widehat{t}) \geq z]$. Consider the non-negative measures $\widehat{\mathrm{g}}^{z}(x, d y)$ on $\mathbb{S} \cap(-\infty, z)$ given by

$$
\widehat{\mathrm{g}}^{z}(x, d y)=\mathbf{P}_{x}\left(\rho<\tau^{z}, \xi(\rho) \in d y\right) .
$$

A convenient way to interpret $\widehat{\mathcal{G}}^{z}(x, d y)$ is to represent the pair $(\xi, \rho)$ in terms of the time-changed process $\widehat{\xi}$. Process $\widehat{\xi}$ is determined by the generator $\widehat{\mathfrak{G}}=\frac{1}{\lambda(x)} \mathfrak{F}$, where $\mathbb{G}$ is the generator of $\xi$. Consider the map $\mathbb{A}: \Omega_{x} \rightarrow \Omega_{x}$, with $\mathbb{A} \omega(t)=\omega\left(t^{\prime}\right)$, where $t^{\prime}$ is the (unique) solution to the equation

$$
t-\int_{0}^{t^{\prime}} \lambda(\omega(s)) d s .
$$

A transforms the pair $\left(\xi_{x}, \rho_{x}\right)$ into $\left(\widehat{\xi}_{x}, \widehat{\rho}\right)$, where $\hat{\rho}$ has an exponential distribution with mean 1 and is independent of process $\widehat{\xi}$. In other words, process $\widehat{\xi}$ spends its 
lifetime at constant rate 1 . The distribution of $\widehat{\xi}$ with the starting point $x$ is denoted by $\widehat{\mathbf{P}}_{x}$. Then

$$
\widehat{\mathscr{S}}^{z}(x, d y)=\int_{0}^{\infty} d t e^{-t} \widehat{\mathbf{P}}_{x}\left(t<\tau^{z}, \widehat{\xi}(t) \in d y\right), \quad x<z .
$$

The above argument shows that we can assume, without loss of generality, that function $\lambda \equiv 1$; the general case is reduces to this case by passing to the new generator $\widehat{\mathfrak{G}}$. We therefore omit the symbol $\widehat{ }$ from the notation. Observe that at this stage we do not assume the IM process $\xi$ to be strong Markov. Our first sufficient condition is:

There exists a function $f^{0}$ on $\mathbb{S}$ satisfying (1.6) and a monotone sequence $\left\{z_{n}\right\} \subseteq \mathbb{R}$ such that $\lim _{n \rightarrow \infty} z_{n}=\infty$ and

$$
f^{0}(x) \geq \mathbf{P}_{x}\left(\rho \geq \tau^{z} n\right) \alpha\left(z_{n}\right)+\int_{\left(-\infty, z_{n}\right)} \mathcal{G}^{z}(x, d y) f^{0}(y) \kappa(y)
$$

where $\alpha(z)=\inf \left[f^{0}(y): y \geq z\right]$.

$$
\left(-\infty, z_{n}\right)
$$

This condition is called Condition S1.

Theorem 2.1: $\quad$ Suppose that Condition S1 hold. Then the BM process $\Xi$ is bounded.

Observe that passing formally to the limit $z_{n} \rightarrow \infty$ in (2.3) gives the following condition called below Condition $\mathrm{N}$ :

There exists a function $f^{0}$ on $\mathbb{S}$ satisfying (1.6) such that

$$
f^{0}(x) \geq \int \mathrm{g}(x, d y) f^{0}(y) \kappa(y)
$$

Here, and below,

$$
\mathcal{G}(x, d y)=\mathbf{P}_{x}(\xi(\rho) \in d y)
$$

$\mathcal{G}(x, d y)$ may be interpreted as a Green measure of process $\xi . \mathrm{N}$ is sufficient for quasiboundedness:

Theorem 2.2: If Condition $\mathrm{N}$ holds then BM process $\Xi$ is quasibounded.

A slightly more restrictive condition that $\mathrm{N}$ is sufficient for the boundedness of a strong branching Markov process. Namely,

There exists a function $f^{0}$ on $\mathbb{S}$ satisfying (1.6) such that

$$
f^{0}(x)=\int \mathfrak{g}(x, d y) f^{0}(y) \kappa(y) .
$$

This condition is called below Condition S2.

Theorem 2.3: If Condition S2 holds and process $\xi$ is strong Markov then BM process $\Xi$ is bounded.

2.2 However, the main role of Condition $\mathrm{N}$ is that it in some cases is necessary for the quasiboundedness (and hence for the boundedness) of a BM process. For this we need additional assumptions about process $\xi$. More precisely, we again work with measures $\mathrm{G}(x, d y)$. The assumptions are as follows:

(i) There exists a point $x_{0} \in \mathbb{S}$ such that for each $x \in \mathbb{S}$ measure $\mathfrak{G}(x, d y)$ is absolutely continuous relatively to $\mathrm{g}\left(x_{0}, d y\right)$.

(ii) The Radon-Nikodym derivative

$$
g_{0}(x, y)=\frac{\mathfrak{G}(x, d y)}{\mathcal{G}\left(x_{0}, d y\right)}
$$


is such that either of the following two conditions holds: $a$ ) for each bounded interval $J \subset \mathbb{R}$ with $J \cap \mathbb{S} \neq \emptyset$, the family $\left\{g_{0}(\cdot, y), y \in \mathbb{R}\right\}$ of functions $x \in J \cap \mathbb{S} \mapsto g_{0}(x, y)$ is uniformly bounded and equicontinuous (and hence compact in $C(J \cap \mathbb{S})$ ); $b$ ) for each $x \in \mathbb{S}$ the function $y \in \mathbb{S} \mapsto g_{0}(x, y)$ is continuous and has finite limits as $y \rightarrow \pm \infty$ (which may depend on $x$ ).

(iii) $\lim _{R \rightarrow \infty}\left(\sup _{x \in \mathbb{S}}(\mathrm{g}(x,(-\infty, x-R]))=0\right.$.

We say that the neighborhood of infinity is not accessible from point $x \in \mathbb{S}$ (in process $\xi$ ) if there exists $z$ such that $\mathbf{P}_{x}\left(\tau^{z}<\infty\right)=0$.

Theorem 2.4: If a BM process $\Xi$ is quasibounded and the corresponding IM process obeys $(i),(i i)$ and (iii), then Condition $\mathrm{N}$ holds. Furthermore, function $f^{0}$ figuring in Condition $\mathrm{N}$ vanishes at any point $x \in \mathbb{S}$ from which the neighborhood of infinity is not accessible.

Throughout this section we use the following notation

$$
F(x, z)=\mathscr{\Phi}_{x}(M<z), \tilde{F}(x, z)=\mathscr{\Phi}_{x}(\tilde{M}<z), \tilde{F}^{c}(x, z)=1-\tilde{F}(x, z) .
$$

$\psi(y, \cdot)$ denotes the moment-generating function of the number of offspring produced at point $y: \psi(y, v)=\sum_{j} K(y, j) v^{j}, y \in \mathbb{R}, 0 \leq v \leq 1$. The symbol $\square$ is used in the sequel for marking the end of the proof.

2.3 Proof of Theorem 2.1: Given $z \in \mathbb{R}$, function $F(\cdot, z)$ gives the maximal solution to the nonlinear integral equation

$$
F(x, z)=1(x<z) \int_{(-\infty, z)} \mathcal{g}^{z}(x, d y) \psi(y, F(y, z)), x \in \mathbb{S},
$$

in the class $\mathcal{U}_{z}$ of functions $u$ on $\mathbb{S}$ bounded by 0 and 1 and satisfying the condition $u(x)=0$ for $x \geq z$. Introduce the operator $\Lambda_{z}$ that represents the RHS of (2.7),

$$
\Lambda_{z} u(x)=1(x<z) \int \mathrm{g}^{z}(x, d y) \psi(y, u(y)), u \in \mathcal{U}_{z}
$$

and write $(2.7)$ as $F(\cdot, z)=\left(\Lambda_{z} F\right)(-\infty, z)$. Operator $\Lambda_{z}$ preserves the pointwise inequality; if $u_{1} \geq u_{2}$ then $\Lambda_{z} u_{1} \geq \Lambda_{z} u_{2}$. Hence, it suffices to prove that there exists a sequence of functions $u_{0 n} \in \mathcal{U}_{z_{n}}$ such that $\Lambda_{z_{n}} u_{0 n} \geq u_{0 n}$ and $\lim _{n \rightarrow \infty} u_{0 n}(x)=1$ for each $x \in \mathbb{S}$. Here $z_{n}$ is the sequence figuring in Condition $\mathrm{S} 1$.

Set

$$
u_{0 n}(x)=\max \left[0,1-\frac{f^{0}(x)}{\alpha\left(z_{n}\right)}\right],
$$

where $f^{0}$ is the function figuring in Condition $\mathrm{S} 1$ (as $f(x) \rightarrow \infty$ as $x \rightarrow \infty$, we can assume that $\left.\alpha\left(z_{n}\right)>0\right)$. Observe that $\psi(y, \cdot)$ is convex and $\kappa(y)=(\partial / \partial u) \psi(y, u)$ at $u=1$. This yields

and hence

$$
\psi(y, u) \geq 1+(u-1) \kappa(y)
$$

$$
\Lambda_{z} u_{0 n}(x) \geq 1\left(x<z_{n}\right) \int_{\left(-\infty, z_{n}\right)} \mathrm{g}^{z} n(x, d y)\left(1-\kappa(y)\left(1-u_{0 n}(y)\right)\right)
$$




$$
\geq 1\left(x<z_{n}\right) \int_{\left(-\infty, z_{n}\right)} \mathrm{g}^{z} n(x, d y)\left(1-\kappa(y) \frac{f^{0}(y)}{\alpha\left(z_{n}\right)}\right)
$$

Thus it suffices to prove the bound

$$
\int_{\left(-\infty, z_{n}\right)} \mathrm{g}^{z} n(x, d y)\left(1-\kappa(y) \frac{f^{0}(y)}{\alpha\left(z_{n}\right)}\right) \geq 1-\frac{f^{0}(x)}{\alpha\left(z_{n}\right)}
$$

But from (2.3) we obtain

$$
\begin{gathered}
1-\frac{f^{0}(x)}{\alpha\left(z_{n}\right)} \leq 1-\mathbf{P}_{x}\left(\rho \geq \tau^{z} n\right)-\int_{\left(-\infty, z_{n}\right)} \mathrm{g}^{z} n(x, d y) \frac{f^{0}(y)}{\alpha\left(z_{n}\right)} \kappa(y) \\
=\int_{\left(-\infty, z_{n}\right)} \mathrm{g}^{z}{ }^{n}(x, d y)-\int_{\left(-\infty, z_{n}\right)} \mathrm{g}^{z}(x, y) \frac{f^{0}(y)}{\alpha\left(z_{n}\right)} \kappa(y) .
\end{gathered}
$$

The proof of Theorem 2.2 follows the same idea as the proof for Theorem 2.1, and we omit it from this paper.

Proof of Theorem 2.3: Set

$$
G_{\mathrm{ex}} \varphi(x)=\int \mathcal{G}(x, d y) \varphi(y)=\mathbf{E}_{x}\left(\int_{0}^{\infty} e^{-t} \varphi(\xi(t)) d t\right) .
$$

We consider $G_{\mathrm{ex}}$ as an operator defined on the domain $D\left(G_{\mathrm{ex}}\right)$ consisting of functions $f$, for which the integrals absolutely converge for $\mathbf{P}_{x}$-almost all trajectories and the expectation exists for each $x \in \mathbb{S}$. $G_{\mathrm{ex}}$ may be considered as an extended Green operator of process $\xi$.

Let $\tau$ be an arbitrary Markov moment. By the strong Markov property of $\xi$, for any $\varphi \in D\left(G_{\mathrm{ex}}\right)$

$$
G_{\mathrm{ex}} \varphi(x)=\mathbf{E}_{x}\left(\int_{0}^{\tau} e^{-t} \varphi(\xi(t)) d t+e^{-\tau} f(\xi(\tau))\right)
$$

where $f=G_{\text {ex }} \varphi$. Choose an arbitrary $z$ and let $\tau^{z}$ be, as before, the time when $\xi$ hits $[z, \infty)$. Consider the function $\varphi_{z}$ :

$$
\begin{aligned}
\varphi_{z}(x) & =\varphi(x), \text { if } x<z, \\
& =f(x), \text { if } x \geq z .
\end{aligned}
$$

Denote again $\xi^{z}(t)=\xi\left(t \wedge \tau^{z}\right)$. Process $\xi^{z}$ is now Markov; we can introduce the corresponding extended Green operator $G_{\mathrm{ex}}^{z}$. Then

$$
\begin{aligned}
& G_{\mathrm{ex}}^{z} \varphi_{z}(x)=\mathbf{E}_{x}\left(\int_{0}^{\infty} e^{-t} \varphi_{z}\left(\xi^{z}(t)\right) d t\right) \\
& =\mathbf{E}_{x}\left(\int_{0}^{\tau} e^{-t} \varphi(\xi(t)) d t+e^{-\tau^{z}} f\left(\xi\left(\tau^{2}\right)\right)\right) .
\end{aligned}
$$


Comparing (2.10) with (2.9), we obtain that $G_{\mathrm{ex}}^{z} \varphi_{z}(x)=G_{\mathrm{ex}} \varphi(x)$. Take $\varphi=\kappa f^{0}$, then by $(2.8), G_{\mathrm{ex}}^{z}\left(\kappa f^{0}\right)_{z}=f^{0}$, where

$$
\begin{aligned}
\left(\kappa f^{0}\right)_{z}(x) & =\kappa(x) f^{0}(x), \text { if } x<z, \\
& =f^{0}(x), \text { if } x \geq z .
\end{aligned}
$$

As before, let $\alpha(z)=\inf [f(x): x \geq z]$. Set

$$
\begin{aligned}
h(x) & =\varphi_{z}(x), \text { if } x<z, \\
& =\alpha(z), \text { if } x \geq z,
\end{aligned}
$$

then $h \leq \varphi_{z}$, so $f^{0}=G_{\mathrm{ex}}^{z} h$. But the RHS coincides with the RHS of (2.3). Hence, Condition S1 holds.

Proof of Theorem 2.4: We assume that $\lim _{z \rightarrow \infty} \tilde{F}^{c}(x, z)=0$. Furthermore,

$$
\begin{gathered}
\tilde{F}^{c}(x, z)=\int \mathcal{G}(x, d y) \tilde{\psi}\left(y, \widetilde{F}^{c}(y, z)\right) \\
=\int \mathcal{g}\left(x_{0}, d y\right) g_{0}\left(x_{0}, y\right) \tilde{\psi}\left(y, \tilde{F}^{c}(y, z)\right), x \in \mathbb{S}, z \in \mathbb{R} .
\end{gathered}
$$

Here, $\tilde{\psi}(y, v)=1-\psi(y, 1-v), y \in \mathbb{R}, 0 \leq v \leq 1$; the last equality in (2.11) uses assumption $(i)$. Set

$$
H(x, z)=\frac{\tilde{F}^{c}(x, z)}{\tilde{F}^{c}\left(x_{0}, z\right)} .
$$

First, assume that condition (iia) holds. Then from (iia) it follows that the family of functions $\{H(\cdot, z), z \in \mathbb{R}\}$ is uniformly bounded and equicontinuous on $J \cap \mathbb{S}$ for any bounded interval $J$. Thus, there is a sequence of points $z_{n} \rightarrow \infty$ such that for any $x \in \mathbb{S}$ there exists the limit

$$
\lim _{n \rightarrow \infty} H\left(x, z_{n}\right)=f^{0}(x) \geq 0
$$

Now assume that condition (iib) holds. Consider the family of measures $\left\{\widetilde{\mathfrak{G}}_{z}, z \in \mathbb{R}\right\}$ on $\mathbb{S}$ defined by

$$
\tilde{\mathrm{G}}_{z}(d y)=\frac{\widetilde{\psi}\left(y, \tilde{F}^{c}(y, z)\right)}{\widetilde{F}^{c}\left(x_{0}, z\right)} \mathrm{G}\left(x_{0}, d y\right)
$$

From (2.11) (with $x=x_{0}$ ) it follows that $\int \widetilde{\mathscr{G}}_{z}(d y)=1$. Consider the Banach space $E$ $(=E(\mathbb{S})$ ) of continuous bounded functions $f: \mathbb{S} \rightarrow \mathbb{R}$ having finite limits at $\pm \underset{\sim}{\sim}$. Note that $E$ is separable. Measure $\widetilde{\mathscr{G}}_{z}$ determines a linear functional $\widetilde{\mathscr{G}}_{z}(\varphi)=$ $\int \widetilde{\mathscr{G}}_{z}(d y) \varphi(y), \quad \varphi \in E$, with norm 1. In view of the weak compactness of the unit sphere in the dual space $E^{*}$, there exists a sequence $z_{n} \rightarrow \infty$ such that $\mathcal{G}_{z}(\varphi)$ converges, as $n \rightarrow \infty$, for all $\varphi \in E$. According to condition $(i i b)$, the function $y \mapsto g_{0}(x, y)$ belongs to $E$ for each $x \in \mathbb{S}$. Therefore, the sequence of values

$$
\widetilde{\mathfrak{g}}_{z_{n}}\left(g_{0}(x, y)\right)=\int g_{0}(x, y) \frac{\tilde{\psi}\left(y, \tilde{F}^{c}\left(y, z_{n}\right)\right)}{\tilde{F}^{c}\left(x_{0}, z_{n}\right)} \mathfrak{g}\left(x_{0}, d y\right)
$$

converges for all $x \in \mathbb{S}$. But (2.14) coincides with (2.12). Hence, the limit (2.13) 
exists.

Having established (2.13), observe that $\lim _{v \rightarrow 0} \frac{1}{\bar{v}} \tilde{\psi}(y, v)=\kappa(y)$. From Fatou's Lemma we deduce that $f^{0}$ obeys (2.4). By construction, $f^{0}$ vanishes at each point from which the neighborhood of infinity is not accessible. It remains to check that $\lim _{x \rightarrow \infty} f^{0}(x)=\infty$. As $1<\kappa_{0} \leq \kappa($ see $(1.1))$,

$$
f^{0}(x) \geq \kappa_{0} \int_{\mathbb{S}} \mathrm{g}(x, d y) f^{0}(y) .
$$

As before, set $\alpha(z)=\inf _{y \geq z} f^{0}(y)$; clearly, $\alpha$ is a non-decreasing function. Using assumption ( $i i i$ ), find $R>0$ and $r>1$ such that $\mathbf{P}_{x}(\xi(\rho)>x-R) \geq \frac{r}{\kappa_{0}}$ for all $x \in \mathbb{R}$. Now fix an $u \in \mathbb{R}$ and let $x$ be $\geq u+R$. According to (2.15),

$$
\begin{gathered}
f^{0}(x) \geq \kappa_{0} \mathcal{G}(x, d y) f^{0}(y) \\
\quad \kappa_{0} \alpha(x-R, \infty) \\
\geq r \alpha(x-R) \geq r \alpha(u) .
\end{gathered}
$$

Thus, $\alpha(u+R) \geq r \alpha(u)$. Deduce that $\lim _{k \rightarrow \infty} \alpha(u+k R)=\infty$.

Remark 2.5: Observe that to establish the relation $\lim _{x \rightarrow \infty} f^{0}(x)=\infty$ it suffices to use (2.4) and assumption (iii).

Remark 2.6: Assumption (iib) may be replaced by a weaker condition that for each $x \in \mathbb{S}$ the function $y \in \mathbb{S} \mapsto g_{0}(x, y)$ is bounded and the family of these functions (indexed by $x \in \mathbb{S}$ ) forms a separable set in the space of bounded Borel functions on $\mathbb{S}$ with supremum norm.

\section{Conditions of Boundedness for the BD}

3.1 In Section 3 we deal with a BD, assuming that the IM process $\xi$ is a diffusion, in the sense given in Itô and McKean [11]), on a set $\mathbb{S}$, which is either the line $\mathbb{R}$ or the "extended" half-line $\overline{\mathbb{R}}_{+}=\{\star\} \cup[0, \infty)$, with a singular (or boundary) point at 0 . By $\mathfrak{5}$ we understand here the "global" generator of process $\xi$, again in the sense of [11]; this means that $\mathbb{S}$ acts on a subset $D(\mathfrak{S}) \subset D_{\xi}$. Here $D_{\xi}\left(=D_{\xi}(\mathbb{S})\right)$ is the space of bounded functions $\mathbb{S} \mapsto \mathbb{R}$, left/right continuous at left/right regular points of $\xi$ and with $f(\star)=0$ (see [11], Chapter 3 , Section 3.6, from which we borrow our notation). More precisely, $D(\mathfrak{G})$ is the image of $D_{\xi}$ under the Green operators

$$
G_{\alpha}: f \in D_{\xi} \mapsto \int_{0}^{\infty} e^{-\alpha t} \mathbf{E}_{x} f(\xi(t)) d t, \alpha>0,
$$

associated with $\xi$, and $\mathfrak{G}$ is defined by $\mathfrak{G}=I-G^{-1}$ where $G=G_{1}$. An important role in our analysis is again played by the Green measures $\mathcal{G}(x, d y)$ and $\mathcal{G}^{z}(x, d y)$ of process $\xi$ and its stopped version $\xi^{z}$ and by their generators $\mathfrak{G}$ and $\mathfrak{F}^{z}$ understood in the aforementioned sense. Observe that

$$
G f(x)=\int g(x, d y) f(y), f \in D_{\xi}
$$


As in the general case, we start with sufficiency. Assume that function $f_{\sim}$ and point $x \in \mathbb{R}$ are such that there exists a neighborhood $U$ of $x$ and a function $\widetilde{f}_{U} \in$ $D(\mathfrak{S})$ coinciding with $f$ on $U \cap \mathbb{S}$. We use the locality theorem for the generator $\mathfrak{S}$ (see the theorem from [11], Chapter 3, Section 3.7, p. 100). According to the locality theorem, value $\mathfrak{5} \widetilde{f}_{U}(x)$ does not depend on the choice of $U$ and $\widetilde{f}_{U}$. We will denote it by $\mathfrak{G}_{\mathrm{lo}} f(x)$. We agree that the domain $D\left(\mathfrak{G}_{\mathrm{lo}}\right)$ of operator $\mathfrak{G}_{\mathrm{lo}}$ consists of all functions $f$ for which the value $\mathfrak{G}_{\mathrm{lo}} f(x)$ is determined, by the above construction, for any $x \in \mathbb{S}$. Furthermore, given a function $f: \mathbb{S} \mapsto \mathbb{R}$, we say that it has property A (relative to $\mathfrak{G})$ if for any $b$ large enough, there exists a function $\tilde{f}_{b} \in D(\mathfrak{F})$ such that $f(x)=$ $\tilde{f}_{b}(x)$ for $x \leq b, x \in \mathbb{S}$. If $f$ has property $\mathrm{A}$ then for any $x \in \mathbb{S}$ and $b>x, b$ large enough, the value $\mathfrak{S} \widetilde{f}_{b}(x)=\mathfrak{S}_{\mathrm{lo}} f(x)$ is determined in a unique way.

Consider the following condition called Condition S3:

There exists a function $f^{0}$ on $\mathbb{S}$ having property A such that relations (1.6), (1.7) hold.

Theorem 3.1: For a BD process $\Xi$, Condition $\mathrm{S} 3$ is sufficient for $\mathrm{S} 1$ and therefore for boundedness.

Proof: Take $z \in \mathbb{S}$ large enough, so that for any $b>z$ function $\tilde{f}_{b}^{0}$ figuring in property A exists. Consider the stopped diffusion IM process $\xi^{z}(t)=\xi\left(t \wedge \tau^{z}\right)$, where $\tau^{z}$ is the time of hitting point $z$ by process $\xi$. Let $G^{z}$ denote the Green operator of $\xi^{z}$ which acts on $D_{\xi^{z}}$, the space of functions on $\mathbb{S} \cap(-\infty, z]$ associated with $\xi^{z}$. Then the domain $D\left(\mathfrak{G}^{z}\right)$ of the generator $\mathfrak{G}^{z}$ coincides with the image of $D_{\xi^{z}}$ under $G^{z}$ and if $h=G^{z} f$ then $\mathfrak{5}^{z} h=h-f$. (See again [11], Chapter 3, Section 3.6.) For any $\alpha \in D_{\xi_{z}}$

$$
G^{z} \varphi(x)=\mathbf{P}_{x}\left(\tau^{z} \leq \rho\right) \varphi(z)+\int_{(-\infty, z)} \mathfrak{S}^{z}(x, d y) \varphi(y), \quad x \leq z .
$$

Take the functions $\tilde{f}_{b}^{0}, b>z$, figuring in property $\mathrm{A}$; their restrictions to $(-\infty, z]$ coincide with the restriction of $f^{0}$ to $(-\infty, z]$. We agree to use here the same symbol for a function and its restriction to $(-\infty, z]$; with this agreement $f^{0}$ and $\tilde{f}_{b}^{0}$ belong to $D\left(\mathfrak{S}^{z}\right)$ and moreover, $\mathfrak{S}^{z} f^{0}(x)=\mathfrak{S}^{z} \widetilde{f}_{b}^{0}(x), x<z$ (see [11], Chapter 3, Section 3.9, p. 103).

Observe that $\mathfrak{G}^{z} f^{0}(z)=0$. Therefore, by (1.7),

$$
\mathfrak{S}_{\mathrm{lo}} f^{0}(x)=\mathfrak{G}^{z} f^{0}(x)+(1-\kappa(z)) f(z) \mathbf{1}_{z}(x), \quad x \leq z,
$$

where $\mathbf{1}_{z}(x)=\mathbf{1}(x=z)$. Again by $(1.7), f^{0}=\mathfrak{F}_{\mathrm{lo}} f^{0}+\kappa f^{0}$. Hence,

$$
\left.f^{0}(x)=\kappa(x) f^{0}(x)+\mathfrak{G}^{z} f^{0}(x)+(1-\kappa)\right) f^{0}(z) \mathbf{1}_{z}(x), x \leq z .
$$

Applying operator $G^{z}$ to (3.2) yields

$$
G^{z} f^{0}(x)=G^{z} \kappa f^{0}(x)+G^{z} \mathfrak{S}^{z} f^{0}(x)+(1-\kappa(z)) f^{0}(z) G^{z} \mathbf{1}_{z}(x), \quad x \leq z .
$$

As $G^{z} \mathfrak{S}^{z} f^{0}=G^{z} f^{0}-f^{0}$ and $G^{z} 1(x)=\mathbf{P}_{x}\left(\tau^{z} \leq \rho\right), x \leq z$, this gives, after an easy calculation,

$$
f^{0}(x)=G^{z} \kappa f^{0}(x)+(1-\kappa(z)) f^{0}(z) \mathbf{P}_{x}\left(\tau^{z} \leq \rho\right), \quad x \leq z .
$$


Substituting $\varphi$ equal to $\kappa f^{0}$ (more precisely, the restriction of $\kappa f^{0}$ to $(-\infty, z]$ ) in (3.1) yields

Thus,

$$
G^{z} \kappa f^{0}(x)=\kappa(z) f^{0}(z) \mathbf{P}_{x}\left(\tau^{z} \leq \rho\right)+\int_{(-\infty, z)} \mathcal{G}^{z}(x, d y) \kappa(y) f^{0}(y), x \leq z .
$$

$$
f^{0}(x)=\mathbf{P}_{x}\left(\tau^{z} \leq \rho\right) f^{0}(z)+\int_{(-\infty, z)} \mathrm{g}^{z}(x, d y) \kappa(y) f^{0}(y) .
$$

As $\alpha(z) \leq f^{0}(z),(3.4)$ leads to

which is nothing but $(2.3)$.

$$
f^{0}(x) \geq \mathbf{P}_{x}\left(\tau^{z} \leq \rho\right) \alpha(z)+\int_{(-\infty, z)} \mathcal{g}^{z}(x, d y) \kappa(y) f^{0}(y)
$$

3.2 A separate question is how to check property A. We discuss this question for the two cases: (a) a smooth BD on $\mathbb{R}$ without boundary points; and (b) a smooth BD on $\overline{\mathbb{R}}+$ with a unique Feller boundary point at 0 . That is, we assume that the operator $\mathfrak{G}_{10}$ is a differential operator

$$
\mathfrak{\Im}_{\mathrm{lo}} f(x)=\frac{\sigma^{2}(x)}{2} f^{\prime \prime}(x)+a(x) f^{\prime}(x) .
$$

Here, functions $a, \sigma$ obey $a_{0} \leq a \leq a_{1}, \sigma_{0} \leq \sigma \leq \sigma_{1}$, where $a_{i}, \sigma_{i}$ are constants, $a_{0}, a_{1} \in \mathbb{R}$ and $\sigma_{0}>0$. We also assume that $a, \sigma \in C^{2}(\mathbb{R})$ in case $(a)$ or $C^{2}\left(\mathbb{R}_{+}\right)$in case $(b)$. In the first case domain $D\left(\mathfrak{G}_{\mathrm{lo}}\right)=C^{2}(\mathbb{R})$; in the second, it consists of the functions $f \in C^{2}\left(\mathbb{R}_{+}\right)$satisfying (3.6). Furthermore, by using integration by parts, it is possible to check that in the first case domain $D(\mathfrak{F})$ of the global generator $\mathbb{5}$ consists of the bounded functions $f \in C^{2}(\mathbb{R})$ for which (3.5) determines a bounded function on $\mathbb{R}$. [Observe that the space $D_{\xi}$ in this case coincides with the set of bounded functions belonging to $C^{0}(\mathbb{R})$.] Similarly, in the second case, $D(\mathbb{G})$ consists of the bounded functions $f$ on $\overline{\mathbb{R}}_{+}$, for which $f(\star)=0, f \in C^{2}\left(\mathbb{R}_{+}\right)$, the RHS of (3.5) determines a bounded function on $\mathbb{R}_{+}$, and the boundary condition

$$
p_{1} f(0)-p_{2} f^{\prime}(0)+p_{3}\left(\frac{\sigma(0)^{2}}{2} f^{\prime \prime}(0)+a(0) f^{\prime}(0)\right)=0
$$

holds for some fixed $p_{i}, i=1,2,3$, satisfying $p_{i} \geq 0 ; p_{1}+p_{2}+p_{3}=1$. The action of (5) on $D(\mathfrak{G})$ is given by the RHS of (3.5).

We also assume (in addition to $(1.1)$ and $(1.2)$ ) that $\kappa \in C^{0}(\mathbb{R})$ or $C^{0}\left(\mathbb{R}_{+}\right)$.

Theorem 3.2: Under the above conditions on functions $a, \sigma$ and $\kappa$, if a function $f^{0}$ obeys (1.6) and (1.7), then it has property A. Thus, Condition CD implies Condition S3.

Proof: Consider the case of a diffusion on $\mathbb{R}$. Suppose that $f^{0}$ satisfies the assumptions of Theorem 3.2. Observe that, due to the maximum principle, $f^{0}$ cannot have a local minimum on $\mathbb{R}$; hence, in view of (1.6), it is monotone nondecreasing, and therefore bounded on any half-line $(-\infty, b]$. Owing to (1.7), the function

$$
x \mapsto \frac{\sigma^{2}(x)}{2} f^{0^{\prime \prime}}(x)+a(x) f^{0^{\prime}}(x)
$$

is also bounded on any half-line $(-\infty, b]$. It is clear that we can prolong $f^{0}$ from 
$(-\infty, b]$ to the whole $\mathbb{R}$ so that the resulting function (as before, denoted by $\tilde{f}_{b}^{0}$ ) belongs to $C^{2}(\mathbb{R})$, is bounded and gives rise to the function

$$
x \mapsto \frac{\sigma^{2}(x)}{2} \tilde{f}_{b}^{0^{\prime \prime}}(x)+a(x) \tilde{f}_{b}^{0^{\prime}}(x)
$$

which is also bounded on $\mathbb{R}$. Thus $\tilde{f}_{b}^{0} \in D(\mathfrak{F})$, and $f^{0}$ has property A.

The same argument works for the case of the diffusion on $\overline{\mathbb{R}}+$.

3.3 In Sections 3.3-3.6 we prove that Criterion CD, in the case of a BD process, is necessary for quasiboundedness (and therefore, boundedness). We again assume that the IM process $\xi$ is a smooth diffusion, either on $\mathbb{R}$ or on $\overline{\mathbb{R}}_{+}$, with a Feller boundary point at 0 .

Theorem 3.3: Suppose that a quasibounded BD process $\Xi$ satisfies the conditions of Section 3.2. Then assumptions (i)-(iii) of Section 2.2 and Condition CD of Section 1.1 hold true.

Proof: We begin with the case of a smooth diffusion on $\mathbb{R}$. The first step is to check that, under the conditions of Theorem 3.3, assumptions (i), (ii) and (iii) hold, and hence the boundedness (or even the quasiboundedness) implies Condition N. We will then deduce Criterion CD from Condition N.

Condition $(i)$ is plain: it is well-known that under the assumptions of Theorem 3.3 , the Green measure $\mathfrak{G}(x, d y), x, y \in \mathbb{R}$, is absolutely continuous, and the RadonNikodym derivative $g(x, y)=\mathfrak{S}(x, d y) / d y$ is the Green function for operator $I-\mathfrak{S}_{\mathrm{lo}}$. Function $g$ is strictly positive and continuous in $x, y$ and admits the representation

$$
\begin{aligned}
g(x, y) & =\zeta_{2}(y) \eta_{1}(x), \quad x \leq y \\
& =\zeta_{1}(y) \eta_{2}(x), \quad y \leq x
\end{aligned}
$$

Here $\eta_{i}$ and $\zeta_{i}, i=1,2$, belong to $C^{2}(\mathbb{R})$ and obey $\eta_{1} \zeta_{2}=\eta_{2} \zeta_{1}, \eta_{i}(x)>0, x \in \mathbb{R}$, $\eta_{1}(-\infty)=\eta_{2}(\infty)=0$, and $\eta_{i}$ satisfy the differential equation $\frac{\sigma^{2}(x)}{2} \eta_{i}^{\prime \prime}(x)+$ $a(x) \eta_{i}^{\prime}(x)=\eta_{i}(x), \quad x \in \mathbb{R}$. Note that these conditions determine functions $\eta_{1}, \eta_{2}$ uniquely, up to constant factors. Functions $\zeta_{1}, \zeta_{2}$ are given by

$$
\zeta_{i}=\frac{2 \eta_{i}}{\sigma^{2} W}
$$

where

$$
W=\eta_{1}^{\prime} \eta_{2}-\eta_{1} \eta_{2}^{\prime}, \quad x \in \mathbb{R}
$$

is the Wronskian. Observe that the above formulas determine function $g$ uniquely.

Furthermore,

$$
\left(\mathfrak{F}_{\mathrm{lo}}\right)_{x} g(x, y)=g(x, y)-\delta(x-y), \quad x, y \in \mathbb{R}
$$

Equation (3.10) is an "extended" differential version of the operator identity $5 G=$ $G-I$ which follows immediately from the definition of the global generator $\mathfrak{G}$. $\left[\left(\mathfrak{G}_{\mathrm{lo}}\right)_{x}\right.$ in the LHS of $(3.10)$ means that operator $\mathfrak{G}_{\text {lo }}$ is applied to variable $x$.] Hence, $g_{0}(x, y)$ in assumption $(i i)$ equals $\frac{g(x, y)}{g\left(x_{0}, y\right)}$. $g_{0}$ is continuous in $x, y$ and, in view of (3.7), does not depend $y$ when $y$ is outside the interval between $x_{0}$ and $x$. Any of the 
conditions (iia) and (iib) then follow immediately.

Finally, assumption (iii) follows from the fact that coefficients $a$ and $\sigma$ are bounded.

So, by Theorem 2.4, if the BD is quasibounded then Condition $\mathrm{N}$ holds. We are now going to deduce Condition CD from $\mathrm{N}$ (in general, functions $f^{0}$ in Conditions $\mathrm{N}$ and CD will be different). Denote the RHS of (2.4) by $\bar{f}^{0}$. Observe that in the case under consideration, $f^{0}(x)>0$ on $\mathbb{R}$. Thus, $\bar{f}^{0}(x)>0$ on $\mathbb{R}$. Applying operator $\mathfrak{G}_{\text {lo }}$ and using (3.10), we obtain

$$
\mathfrak{G}_{\mathrm{lo}} \bar{f}^{0}(x)=\frac{\sigma^{2}(x)}{2} \bar{f}^{0 \prime \prime}(x)+a(x) \bar{f}^{0 \prime}(x)=\bar{f}^{0}(x)-\kappa(x) f^{0}(x) .
$$

According to $(2.4), f^{0}(x) \geq \bar{f}^{0}(x)$ [i.e., $\kappa(x) f^{0}(x) / \bar{f}^{0}(x) \geq \kappa(x)$ ]. Therefore, $\bar{f}^{0}=$ $\int \mathfrak{G}(x, d y) \kappa(y) f^{0}(y) \geq \int \mathfrak{G}(x, d y) \kappa(y) \bar{f}^{0}(y)$. Thus, function $\bar{f}^{0}$ obeys (2.4). As assumption (iii) is fulfilled, we can deduce that $\lim _{x \rightarrow \infty} \bar{f}^{0}(x)=\infty$ (see Remark 2.5).

At this stage, we apply Lemma 3.5 (see below), with $l_{2}(x)=\kappa(x) f^{0}(x) / \bar{f}^{0}(x)-1$ and $l_{2}(x)=\kappa(x)-1$. According to Lemma 3.5 , there exists a positive solution of Equation (1.7) for which (1.6) holds true. This completes the proof of Theorem 3.3 for the case of a smooth diffusion IM process on $\mathbb{R}$.

3.4 Lemma 3.5 is an elementary result from the theory of ordinary differential equations. Despite its transparent character (and short proof), we failed to track down this result in the available literature. The same is true of Lemmas 3.6 and 3.7 below.

Consider a second-order differential equation

$$
y^{\prime \prime}(x)+\alpha(x) y^{\prime}(x)-\beta(x) y(x)=0, \quad x \in \mathbb{R} .
$$

Suppose that coefficients $\alpha$ and $\beta$ are $C^{0}$-functions and $\beta \geq 0$.

Lemma 3.4: Under the above conditions, equation (3.12) possesses a solution $y_{0}$ such that

$$
y_{0}(x) \geq 1, x \in \mathbb{R} .
$$

Proof: If $\beta \equiv 0$, we can take $y_{0} \equiv 1$. Now suppose that $\beta\left(x_{0}\right)>0$ for some $x_{0} \in \mathbb{R}$. Consider the solution of the Cauchy problem for (3.12), with the initial condition

$$
y\left(x_{0}\right)=1, y^{\prime}\left(x_{0}\right)=0
$$

and denote it again by $y_{0}$. Since $\beta\left(x_{0}\right)>0$, function $y_{0}$ has a local minimum at point $x_{0}$. On the other hand, by the maximum principle, the solution of (3.12) cannot have a local positive maximum. Hence, $y_{0}$ obeys (3.13).

Lemma 3.4 is used to prove

Lemma 3.5: Let functions $a$ and $\sigma$ satisfy the conditions of Section 3.2. Assume that $l_{1}$ and $l_{2}$ are two $C^{0}$-functions on $\mathbb{R}$, and $l_{1} \geq l_{2}$. Suppose that there exists a positive solution $g_{1}$ of the equation

$$
\frac{\sigma(x)^{2}}{2} g^{\prime \prime}(x)+a(x) g^{\prime}(x)=-l_{1}(x) g(x), \quad x \in \mathbb{R} .
$$

Then there exists a solution $g_{2}$ of

$$
\frac{\sigma(x)^{2}}{2} g^{\prime \prime}(x)+a(x) g^{\prime}(x)=-l_{2}(x) g(x), \quad x \in \mathbb{R}
$$


with $g_{2} \geq g_{1}$.

Proof: We search for a solution $g_{2}$ of the form $g_{2}(x)=g_{1}(x) y(x)$. Then for function $y$ we have an equation of form (3.12), with

$$
\alpha(x)=\frac{2 a(x)}{\sigma(x)^{2}}+2 z(x), \text { and } \beta(x)=\frac{2\left(l_{1}(x)-l_{2}(x)\right)}{\sigma(x)^{2}},
$$

where $z(x)=(\log g(x))^{\prime}$. By virtue of Lemma 3.4, $y \geq 1$.

3.5 The above argument may be extended to the case where the IM process is a smooth diffusion on $\overline{\mathbb{R}}_{+}=\{\star\} \cup[0, \infty)$, with a Feller boundary point at 0 . The proof of Theorem 3.3 in this case follows the same line. The Green measure $\mathrm{g}(x, d y)$, $x \geq 0$, may now have an atom at 0 , but its restriction to $(0, \infty)$ is absolutely continuous, and the Radon-Nikodym derivative $g(x, y)=\mathfrak{G}(x, d y) / d y$ is positive for $x$, $y>0$. For any $x_{0}>0$, the function

$$
g_{0}(x, y)=\frac{g(x, y)}{g\left(x_{0}, y\right)}, x, y>0
$$

may be continuously prolonged to $\mathbb{R}_{+} \times \mathbb{R}_{+}$(i.e., defined for $x, y \geq 0$ ). We remark that the function on $\mathbb{R}_{+} \times \mathbb{R}_{+}$so defined (we again denote it by $\bar{g}_{0}$ ) coincides with the Radon-Nikodym derivative $\frac{g(x, d y)}{\mathcal{G}\left(x_{0}, d y\right)}$. Furthermore, function $g$ admits, for $x, y>0$, the representation (3.7), where functions $\eta_{1}$ and $\zeta_{i}$ again obey $\eta_{1} \zeta_{2}=\eta_{2} \zeta_{1}$, $\eta_{i}(x)>0, \quad x>0, \quad p_{2} \eta_{1}^{\prime}(0)=\left(p_{1}+p_{3}\right) \eta_{1}(0), \eta_{2}(\infty)=0$, and functions $\eta_{i}$ satisfy the differential equation $\frac{\sigma^{2}(x)}{2} \eta_{i}^{\prime \prime}(x)+a(x) \eta_{i}^{\prime}(x)=\eta_{i}(x), x>0$. As before, functions $\eta_{1}, \eta_{2}$ are determined by these conditions uniquely, up to constant factors, and functions $\zeta_{1}$, $\zeta_{2}$ are given by (3.8) and (3.9). Function $g$ is again determined by the above formulas uniquely and obeys (3.10) for $x, y>0$.

The above properties enable us to repeat the argument and check assumptions (i)-(iii) of Section 2.2 for the case under consideration. Therefore, Condition $\mathrm{N}$ is again valid, and function $f^{0}$ is $>0$ on $(0, \infty)$. What remains is to deduce Condition CD from N. Denoting, as before, the RHS of $(2.4)$ by $\bar{f}^{0}$, we have $\bar{f}^{0}>0$ on $(0, \infty)$ and can write (3.11). Observe that, by virtue of Remark 2.5, we again have $\lim _{x \rightarrow \infty} \bar{f}^{0}(x)=\infty$.

3.6 At this point, we are going to use Lemmas 3.6 and 3.7 which are modified versions of Lemmas 3.4 and 3.5, respectively. [The way these lemmas are used differs for the case $p_{2}>0$ and $p_{2}=0$, see below.]

Lemma 3.6: Let $\alpha$ and $\beta$ be $C^{0}$-functions on $(0, \infty)$ and $\beta \geq 0$. Given $\epsilon>0$, let $y_{\epsilon}$ denote the solution of Equation (3.12) satisfying $y_{\epsilon}(\epsilon)=1, y_{\epsilon}^{\prime}(\epsilon) \geq 0$. Then $y_{\epsilon} \geq 1$ on $[\epsilon, \infty)$.

Proof: The proof of Lemma 3.6 again follows from the maximum principle.

Lemma 3.7: Let functions $a, \sigma$ and l belong to $C^{0}\left(\mathbb{R}_{+}\right)$. Assume that function $f \in C^{2}\left(\mathbb{R}_{+}\right)$is $\geq 0$ on $\mathbb{R}_{+}$and $>0$ on $(0, \infty)$ and satisfies the differential inequality

$$
\frac{\sigma(x)^{2}}{2} f^{\prime \prime}(x)+a(x) f^{\prime}(x) \leq f(x) l(x), \quad x \geq 0 .
$$

Then the function $g$ satisfying the equation 


$$
\frac{\sigma(x)^{2}}{2} g^{\prime \prime}(x)+a(x) g^{\prime}(x)=g(x) l(x), x \geq 0
$$

with the initial data $g(0)=f(0), g^{\prime}(0) \geq f^{\prime}(0)$, obeys $g(x) \geq f(x), x \geq 0$.

Proof: Substitute $g=y f$ in (3.18). Then for the function $y$ we obtain an equation similar to $(3.12)$, with $\alpha(x)=2 a(x) / \sigma(x)^{2}+2(\log f(x))^{\prime}$ and $\beta(x)=$ $2(f(x) l(x)-h(x)) /\left(\sigma(x)^{2} f(x)\right)$, where $h(x)$ denotes the LHS of (3.17). Functions $\alpha$ and $\beta$ satisfy the conditions of Lemma $3.6(\beta(x) \geq 0$ by virtue of (3.17)). Take an $\epsilon>0$ and construct, on $[\epsilon, \infty)$, a solution $y_{\epsilon}(x)$ of $(3.12)$ with initial data $y_{\epsilon}(\epsilon)=1$ and $y_{\epsilon}^{\prime}(e)=\left(g^{\prime}(0)-f^{\prime}(0)\right) / f(\epsilon)$. The function $g_{\epsilon}(x)=y_{\epsilon}(x) f(x)$ gives a solution to (3.18) on $[\epsilon, \infty)$ with initial data $g_{\epsilon}(\epsilon)=f(\epsilon)$ and $g_{\epsilon}^{\prime}(\epsilon)=f^{\prime}(\epsilon)+g^{\prime}(0)-f^{\prime}(0)$. $g_{\epsilon}$ may be prolonged, as a solution to $(3.18)$, to $[0, \infty)$. We obtain a solution of $(3.18)$ with conditions at $x=\epsilon$. Letting $\epsilon \rightarrow 0$ and using the continuity of the solution in the initial condition, we obtain that $g_{\epsilon}(x)$ converges to $g(x), x \geq 0$, as $\epsilon \rightarrow 0$.

According to Lemma 3.6, $y_{\epsilon}(x) \geq 1$ and hence $g_{\epsilon}(x) \geq f(x), x \geq \epsilon$. Thus, $g(x) \geq$ $f(x), x \geq 0$.

To complete the proof of Theorem 3.3, we take the solution of the equation

$$
\frac{\sigma(x)^{2}}{2} g^{\prime \prime}(x)+a(x) g^{\prime}(x)=(1-\kappa(x)) g(x), \quad x \geq 0
$$

with initial data $g(0)=\bar{f}^{0}(0)$ and

$$
\begin{aligned}
g^{\prime}(0) & =\bar{f}^{0 \prime}(0), \text { if } p_{2}=0 \\
& =\bar{f}^{0 \prime}(0)+p_{3} \kappa(0)\left(f^{0}(0)-\bar{f}^{0}(0)\right) / p_{2}, \text { if } p_{2}>0
\end{aligned}
$$

Observe that in the second case $g^{\prime}(0) \geq \bar{f}^{0 \prime}$. By Lemma 3.7 (with $l=1-\kappa$ ), $g(x) \geq$ $\bar{f}^{0}(x), x \geq 0$.

Function $g$ (specified in the preceding paragraph) is an obvious candidate for the role of function $f^{0}$ figuring in Condition CD. It remains to check that it satisfies the boundary conditions (3.6), so that the LHS of (3.19) can be treated as $\mathfrak{S}_{\mathrm{lo}} g(x)$.

If $p_{2}=0$ then $\bar{f}^{0}(0)=0$ (the neighborhood of infinity is not accessible from 0 ). Hence $g(0)=0$ and the LHS of (3.19) vanishes at $x=0$. Therefore, (3.6) holds if we substitute $f(0)$ with $g(0)$. If $p_{2}>0$ then we use the fact that $\bar{f}^{0}$ obeys the boundary condition

$$
p_{2} \bar{f}^{0 \prime}(0)=p_{1} \bar{f}^{0}(0)+p_{3}\left(\frac{\sigma(0)^{2}}{2} \bar{f}^{0 \prime \prime}(0)+a(0) \bar{f}^{0 \prime}(0)\right)
$$

together with the equality

$$
\frac{\sigma(0)^{2}}{2} \bar{f}^{0 \prime \prime}(0)+a(0) \bar{f}^{0 \prime}(0)=\bar{f}^{0}(0)-\kappa(0) f^{0}(0) .
$$

This again leads to $(3.6)$ for $f(0)=g(0)$.

3.7 In Sections 3.8-3.9 we extend the previous discussion to the case of a BDJ process $\Xi$ where IM process $\xi$ is a diffusion on $\overline{\mathbb{R}}_{+}$, with a boundary point at 0 and a jump from 0 to $(0, \infty)$. We retain the assumptions of Section 3.2 on functions $a, \sigma$ and $\kappa$. The boundary condition (3.6) is now replaced by

$$
p_{1} f(0)-p_{2} f^{\prime}(0)+p_{3}\left(\frac{\sigma(0)^{2}}{2} f^{\prime \prime}(0)+a(0) f^{\prime}(0)\right)=\mu \int_{(0, \infty)} p(d y)(f(y)-f(0)) .
$$


As in (3.6), $p_{i} \geq 0, i=1,2,3$, and $\mu \geq 0$ are constants; $p_{1}+p_{2}+p_{3}+\mu=1$.

The domain $D(\mathfrak{G})$ of the "global" generator $\mathfrak{F}$ of process $\xi$ consists the bounded functions $f$ on $\overline{\mathbb{R}}_{+}$, for which $f(\star)=0, f \in C^{2}\left(\mathbb{R}_{+}\right)$, the RHS of (3.5) determines a bounded function on $\mathbb{R}_{+}$, and (3.20) holds. The action of $\mathfrak{G}$, on $D(\mathfrak{F})$, is again given by the RHS of (3.5). The domain $D\left(\mathfrak{G}_{\mathrm{lo}}\right)$ of the operator $\mathfrak{G}_{\text {lo }}$ consists of functions $f \in C^{2}\left(\mathbb{R}_{+}\right)$satisfying (3.20) (with the condition that the integral in the RHS of (3.20) converges absolutely).

Property A and Condition S3, relative to operator $\mathfrak{5}$ defined in the preceding paragraph, are formulated in exactly the same way as in Section 3.1.

Theorem 3.4: For a BDJ process $\Xi$, Condition $\mathrm{S} 3$ is sufficient for $\mathrm{S} 1$ and therefore for boundedness.

Proof: Choose $z$ large enough so that for all $b>z$ there exists the function $\tilde{f}_{b}^{0}$ figuring in property A. Set $\xi^{z}=\xi^{z}\left(t \wedge \tau^{z}\right)$ where $\tau^{z}$ is the first time that process $\xi$ hits the half-line $[z, \infty)$. As $\tilde{f}_{b}^{0} \in D(\mathfrak{\mathcal { S }})$, we have that $\tilde{f}_{b}^{0}=G \varphi$ for some $\varphi \in D_{\xi}$.

Set

$$
\begin{aligned}
\varphi_{z} & =\varphi(z), x<z \\
& =\tilde{f}_{b}^{0}(x), x \geq z .
\end{aligned}
$$

Then, as proved in Section 2.3, $\varphi_{z} \in D_{\xi^{z}}$ and $G^{z} \varphi_{z}=G \varphi=\tilde{f}_{b}^{0}$. Thus, $\tilde{f}_{b}^{0} \in D\left(\mathfrak{G}^{z}\right)$ and $\mathfrak{G}^{z} \tilde{f}_{b}^{0}=\tilde{f}_{b}^{0}-\varphi_{z}$. But then $\mathfrak{S}^{z} \tilde{f}_{b}^{0}=I_{z} \mathfrak{S}^{\tilde{f}_{b}^{0}}=I_{z} \mathfrak{G}_{\mathrm{lo}} f^{0}$, where $I_{z}(x)=1(x<z)$. By virtue of (1.7), we obtain that

$$
I_{z} f^{0}=I_{z} \kappa f^{0}+\mathfrak{5}^{z} \tilde{f}_{b}^{0}
$$

Applying operator $G^{z}$, we have that $G^{z} I_{z} f^{0}=G^{z} I_{z} \kappa f^{0}+G^{z} \tilde{f}_{b}^{0}-\tilde{f}_{b}$. Denote $J_{z}(x)=1-I_{z}(x)=\mathbf{1}(x \geq z)$. As $I_{z} f^{0}=I_{z} \tilde{f}_{b}^{0}$, we obtain that

$$
\tilde{f}_{b}^{0}=G^{z} I_{z} \kappa f^{0}+G^{z} J_{z} \tilde{f}_{b}^{0}
$$

Now calculate function $G^{z} J_{z} \tilde{f}_{b}^{0}$. First, note that both $f^{0}$ and $\tilde{f}_{b}^{0}$ satisfy $(3.20)$, and the LHS of (3.20) for these functions are the same. Therefore,

$$
\int p(d y) J_{z}(y) f^{0}(y)=\int p(d y) J_{z}(y) \tilde{f}_{b}^{0}(y) .
$$

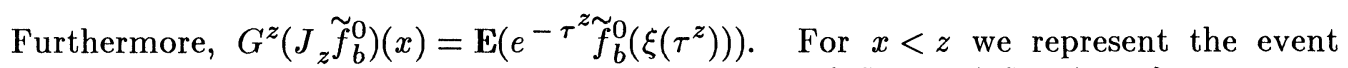
$\left\{\tau^{z} \leq \rho\right\}$ as the disjoint union $A_{0} \cup A_{z}$, where $A_{0}=\left\{\tau^{z} \leq \rho, \xi\left(\tau^{z}-0\right)=0\right\}$ and $A_{z}=$ $\left\{\tau^{z} \leq \rho, \xi\left(\tau^{z}-0\right)=z\right\}$

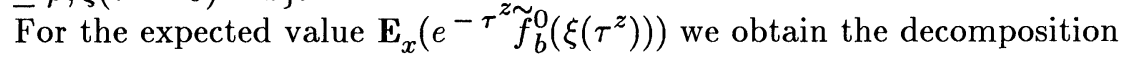

$$
\begin{gathered}
G^{z}\left(J_{z} \tilde{f}_{b}^{0}\right)(x)=\mathbf{P}_{x}\left(A_{0}\right) \mathbf{E}_{x}\left(e^{-\tau^{z} \tilde{f}_{b}^{0}}\left(\xi\left(\tau^{z}\right)\right) \mid A_{0}\right) \\
+\mathbf{P}_{x}\left(A_{z}\right) \mathbf{E}_{x}\left(e^{-\tau^{z} \tilde{f}_{b}^{0}}\left(\xi\left(\tau^{z}\right)\right) \mid A_{z}\right)
\end{gathered}
$$

For the second term in (3.24), we have

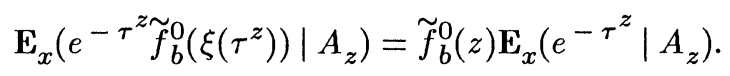


The first term in (3.24) equals

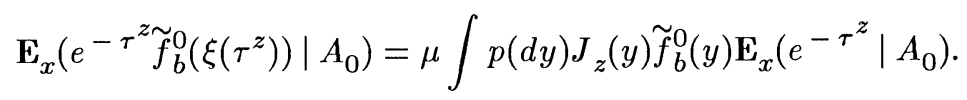

Taking into account (3.23) yields

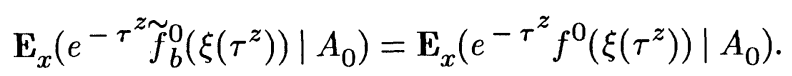

As $\tilde{f}_{b}^{0}(z)=f^{0}(z)$, we have for $x<z$

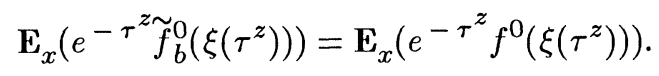

This yields the equality $G^{z}\left(J_{z} \tilde{f}_{b}^{0}\right)(x)=G^{z}\left(J_{z} f^{0}\right)(x), x<z$. Then, by virtue of (3.22),

$$
f^{0}(x)=\tilde{f}_{b}^{0}(x)=G^{z}\left(I_{z} \kappa f^{0}\right)(x)+G^{z}\left(J_{z} f^{0}\right)(x), x<z
$$

As $J_{z}(x) f^{0}(x) \geq J_{z}(x) \alpha(z)$, we obtain

$$
f^{0}(x) \geq G^{z}\left(I_{z} \kappa f^{0}\right)(x)+\alpha(z) \mathbf{P}_{x}\left(\tau^{z} \leq \rho\right), x<z .
$$

The same inequality trivially holds for $x \geq z$. This yields Condition S1.

As before, a separate question is when property A may be verified. Under the above assumptions, the assertion of Theorem 3.2 remains valid also for a BDJ process. The proof of this fact follows the same lines as in Section 3.2. Thus, for the BDJ processes under consideration, Condition CD implies S3 and hence is sufficient for boundedness.

3.8 In this section we analyze the necessary conditions for boundedness of a BDJ process.

Theorem 3.5: Suppose that a quasibounded BDJ process $\Xi$ satisfies the conditions of Section 3.8. Then assumptions ( $i)$, (iib) and (iii) of Section 2.2 and Condition CD of Section 1.1 hold true.

Proof: The proof follows the same argument as that of Theorem 3.3, and we omit some technical detail. The Green measure $\mathrm{g}(x, d y), x \geq 0$, may have an atom at 0 (if $p_{3}>0$ in $(3.20)$ ), but is absolutely continuous on $(0, \infty)$ (even if measure $p$ has atoms). More precisely,

$$
\mathcal{G}(x, d y)=(g(x, y)+b(y) \eta(x)) d y+\alpha \eta(x) \delta_{0}, x \geq 0,
$$

where $\delta_{0}$ is the Dirac delta-measure concentrated at 0 . Functions $g, \eta$ and $b$ and constant $\alpha$ are connected to each other via a system of relations described below. Namely, function $g$ admits the representation (3.7), where functions $\eta_{i}, \zeta_{i}, i=1,2$, belong to $C^{2}(\mathbb{R})$ and obey $\eta_{1} \zeta_{2}=\eta_{2} \zeta_{1}, \quad \eta_{i}(x)>0, \quad x>0, \quad p_{2} \eta_{1}^{\prime}(0)=\left(p_{1}+p_{3}+\mu\right) \eta_{1}(0)$, $\eta_{2}(\infty)=0$, and $\eta_{i}$ satisfy the differential equation $\frac{\sigma^{2}(x)}{2} \eta_{i}^{\prime \prime}(x)+a(x) \eta_{i}^{\prime}(x)=\eta_{i}(x)$, $x>0$. Functions $\eta_{1}, \eta_{2}$ are again determined by these conditions uniquely, up to constant factors, and functions $\zeta_{1}, \zeta_{2}$ are given by (3.8) and (3.9). As before, function $g$ is determined by the above formulas uniquely and obeys (3.10) for $x, y>0$. 
Function $\eta$ in (3.25) coincides with $\eta_{2}$. Constant $\alpha$ has the form $\alpha=p_{3} / d$, where

$$
d=\left(p_{1}+p_{3}+\mu\right) \eta_{2}(0)-p_{2} \eta_{2}^{\prime}(0)-\mu \int_{(0, \infty)} p(d y) \eta_{2}(y)
$$

(the integral $\mu \int_{(0, \infty)} p(d y) \eta_{2}(y)$ is finite and $d>0$ as function $\eta_{2}$ is decreasing). Finally, function $b$ is given by

$$
b(y)=\frac{\mu}{d} \int_{(0, \infty)} p(d z) g(z, y) .
$$

Observe that these relations determine the products $\alpha \eta$ and $b \eta$ in a unique way.

Fix $x_{0}>0$ and set

$$
g_{0}(x, y)=\frac{g(x, y)+\eta_{2}(x) b(y)}{g\left(x_{0}, y\right)+\eta_{2}\left(x_{0}\right) b(y)}, x, y>0 .
$$

By continuation, prolong function $g_{0}$ on $\mathbb{R}_{+} \times \mathbb{R}_{+}$. Then $g_{0}(x, y)$ gives the RadonNikodym derivative $\frac{\mathrm{g}(x, d y)}{\mathrm{g}\left(x_{0}, d y\right)}$. This yields assumption $(i)$.

We now check (iib). By using (3.8) and (3.9), it is easy to check that both functions $g$ and $b$ are continuous in $y$. It remains to verify that for each $x \geq 0$ there exists a finite limit $\lim _{y \rightarrow \infty} g_{0}(x, y)$. According to $(3.7)$, for $y>\max \left[x, x_{0}\right], x \geq 0$,

$$
g_{0}(x, y)=\frac{\eta_{1}(x) \zeta_{2}(y)+\eta_{2}(x) b(y)}{\eta_{1}\left(x_{0}\right) \zeta_{2}(y)+\eta_{2}\left(x_{0}\right) b(y)}
$$

It is clear that it suffices to establish that there exists the (possibly infinite) limit $\lim _{y \rightarrow \infty} \frac{b(y)}{\zeta_{2}(y)}$. Observe that the ratio $\frac{\zeta_{1}(y)}{\zeta_{2}(y)}=\frac{\eta_{1}(y)}{\eta_{2}(y)}$ (see (3.8)) is nondecreasing in $y$. By using (3.7), it is easy to deduce that for each $x \geq 0$, the ratio $\frac{g(x, y)}{\zeta_{2}(y)}$ is also nondecreasing in $y$, and hence $\lim _{y \rightarrow \infty} \frac{g(x, y)}{\zeta_{2}(y)}=\eta_{1}(x)$. Thus the ratio $\frac{b(y)}{\zeta_{2}(y)}$ is nondecreasing, and the above limit exists (and equals $\frac{\mu}{d} \int_{(0, \infty)} p(d z) \eta_{1}(z)$ ).

Assumption (iii), as before, follows from the boundedness of coefficients $a$ and $\sigma$.

Therefore, if a BDJ process $\Xi$ is quasibounded, it satisfies Condition N: there exists a $f^{0}$ obeying (1.6) such that $f^{0} \geq G\left(\kappa f^{0}\right)$. Set $\bar{f}^{0}=G\left(\kappa f^{0}\right)$. Then $\bar{f}^{0}$ belongs to $C^{2}\left(\mathbb{R}_{+}\right)$and satisfies the boundary condition

$$
\left(p_{1}+\mu\right) \bar{f}^{0}(0)-p_{2} \bar{f}^{0 \prime}(0)+p_{3} \mathfrak{G}_{\mathrm{lo}} \bar{f}^{0}(0)=\mu \int_{(0, \infty)} p(d y) \bar{f}^{0}(y) .
$$

In other words, $\bar{f}^{0} \in D\left(\mathfrak{G}_{\mathrm{lo}}\right)$. As in Section 3.3, Remark 2.5 implies that $\lim _{x \rightarrow \infty} \bar{f}^{0}=\infty$. Applying operator $\mathfrak{S}_{\text {lo }}$ yields $\mathfrak{S}_{\mathrm{lo}} \bar{f}^{0}=\bar{f}^{0}-\kappa f^{0} \leq \bar{f}^{0}(1-\kappa)$.

We now want to check that Condition CD holds. Consider the equation

$$
\frac{\sigma(x)^{2}}{2} u^{\prime \prime}(x)+a(x) u^{\prime}(x)=(1-\kappa(x)) u(x), \quad x \geq 0 .
$$

We will show that there exists a solution $u$ to (3.30) satisfying (1.6) such that

$$
\left(p_{1}+\mu\right) u(0)-p_{2} u^{\prime}(0)+p_{3}(1-\kappa(0)) u(0)=\mu \int_{(0, \infty)} p(d y) u(y) \text {. }
$$


In fact, (3.30) has two linearly independent solutions, $u_{1}$ and $u_{2}$. Clearly, there exist constants $c_{1}$ and $c_{2}$ such that the linear combination $u=c_{1} u_{1}+c_{2} u_{2}$ satisfies (3.31). This means that $u \in D\left(\mathfrak{G}_{\mathrm{lo}}\right)$. We first check that $u(0) \neq 0$. Indeed, if $u(0)=0$ then $(3.31)$ becomes

$$
-p_{2} u^{\prime}(0)=\mu \int_{(0, \infty)} p(d y) u(y) .
$$

Suppose, for definiteness, that $u^{\prime}(0)>0$ and write $u$ in the form $u=y \bar{f}^{0}$. Then for function $y$ we have an equation similar to (3.12). As $u(0)=0$ and $u^{\prime}(0)>0$, we have that $y(0)=0$ and $y^{\prime}(0)>0$. Function $y$ cannot have a positive maximum. Thus, $y(x)>0$ and hence $u(x)>0, x>0$. We see that the LHS and RHS of (3.32) are of different signs (the LHS is $\leq 0$ whereas the RHS $>0$ ) which yields a contradiction. Thus, $u(0) \neq 0$.

Function $u$ was determined up to a constant factor. Choose it so that $u(0)=$ $\bar{f}^{0}(0)$. Set $v(x)=u(x)-\bar{f}^{0}(x)$. As $\mathfrak{G}_{\mathrm{lo}} \bar{f}^{0}(x) \leq(1-\kappa(x)) \bar{f}^{0}(x)$, function $v$ satisfies the inequality $\mathfrak{G}_{\mathrm{lo}} v(x) \geq(1-\kappa(x)) v(x)$. As $v(0)=u(0)-\bar{f}^{0}(0)=0$, value $\mathfrak{G}_{\mathrm{lo}} v(0) \geq$ 0 .

From (3.29) and (3.31) we find that

$$
-p_{2} v^{\prime}(0)+p_{3} \mathfrak{G}_{\mathrm{lo}} v(0)=\mu \int p(d y) v(y)
$$

If $v^{\prime}(0) \geq 0$ then $u^{\prime}(0) \geq \bar{f}^{0}(0)$. By virtue of Lemma 3.7, $u(x) \geq \bar{f}^{0}(x), x \geq 0$. Thus, $u$ obeys (1.6).

Now suppose that $v^{\prime}(0)<0$. Then $v(x)<0$ for $x>0$ small enough. On the other hand, as $\mathfrak{S}_{\mathrm{lo}} v(0) \geq 0$, the LHS of $(3.33)$ is positive. Therefore, $\int_{(0, \infty)} p(d y) v(y)>0$. Thus, there exists $x>0$ with $v(x)>0$. Denote by $x^{0}$ the minimal positive point with $v\left(x^{0}\right)=0$. At this point $v^{\prime}\left(x^{0}\right)>0$. Then $u\left(x^{0}\right)=\bar{f}^{0}\left(x^{0}\right)$ and $u^{\prime}\left(x^{0}\right)>\bar{f}^{0 \prime}\left(x^{0}\right)$. Now we use Lemma 3.7 , with point 0 replaced by $x^{0}$. This yields $u(x) \geq \bar{f}^{0}(x), x \geq$ $x^{0}$. Therefore, $u(x)>0, x \geq x^{0}$, and $\lim _{x \rightarrow \infty} u(x)=\infty$. It remains to prove that $u(x) \geq 0$ for $0 \leq x<x^{0}$. Again write $u=y \bar{f}^{0}$. Then $y(0)=1$ (as $\left.u(0)=\bar{f}^{0}(0)\right)$ and $y^{\prime}(0)<0$ (since $v^{\prime}(0)=u^{\prime}(0)-\bar{f}^{0}(0)<0$ ). Function $y$ satisfies $(3.12)$ and hence cannot have a negative minimum. At the same time, the equality $u\left(x^{0}\right)=\bar{f}^{0}\left(x^{0}\right)$ implies that $y_{0}\left(x^{0}\right)=1$. Thus, $y(x) \geq 0$ for $0 \leq x \leq x^{0}$. We can now conclude that $u$ again satisfies (1.6). Thus, Condition CD is fulfilled for $f^{0}=u$.

\section{Conditions of Boundedness for the BJM Processes}

4.1 We again begin with sufficient conditions. A jump Markov process $\xi$ is determined by a function $\mu(x), x \in \mathbb{S}$, and transition probability measure $\pi(x, d y)$, $x, y \in \mathbb{S}$. Throughout Section 4 we assume that

$$
0<\mu_{0} \leq \mu(x) \leq \mu_{1}, \quad x \in \mathbb{S},
$$

where $\mu_{0}<\mu_{1}<\infty$ are constants, and $\mu \in C(\mathbb{S})$. We denote by $T(t), t \geq 0$, the semigroup of operators corresponding to $\xi$ which acts in the space $B(\mathbb{S})$ of bounded Borel functions $f$ on $\mathbb{S}$, with $f(\star)=0$. The generator of the semi-group, $\mathfrak{G}$, is defined on the whole of $B(\mathbb{S})$ by 


$$
\mathfrak{S} f(x)=-\mu(x) f(x)+\mu(x) \int \pi(x, d y) f(y) .
$$

The extended generator, $\mathfrak{S}_{\text {in }}$, is defined on all Borel functions $f$ on $\mathbb{S}$ for which the integral in the RHS of (4.1) exists, and is given again by (4.1). [The notation $\mathfrak{S}_{\text {in }}$ stresses that (4.1) is an integral operator.] The set of such functions is denoted by $D\left(\mathfrak{S}_{\text {in }}\right)$.

Theorem 4.1: If Condition SJ holds then the BJM process $\Xi$ is bounded.

Proof: Set

$$
\Pi f(x)=\int \pi(x, d y) f(y) .
$$

Then $\Pi$ is a linear operator with the domain $D\left(\mathfrak{F}_{\text {in }}\right)$. Let $f^{0}$ be the function from Condition SJ. Observe that $\Pi f^{0}(x)=\left(1-\frac{\kappa(x)-1}{\mu(x)}\right) f^{0}(x)$. Thus, $\Pi f^{0} \leq c f^{0}$ where $c>0$ is a constant. Consider the extended Green operator $G_{\text {ex }}$ of process $\xi$ (see Section 2.3); in this section we denote it by $G_{\text {in }}$. Operator $G_{\text {in }}$ is integral, in Green measure $\mathcal{G}(x, d u)$. If $\theta \in B(\mathbb{S})$, then $\mathfrak{S}_{\text {in }} \theta=\theta-G_{\text {in }}^{-1} \theta$.

We fist show that $f^{0} \in D\left(G_{\text {in }}\right)$. Choose a sequence $f_{n} \in B(\mathbb{S})$ such that $f_{n} \uparrow f^{0}$. Then $\mathfrak{G}_{\text {in }} f_{n} \uparrow \mathfrak{G}_{\text {in }} f^{0}=f^{0}-\kappa f^{0}$. Set $\varphi_{n}=f^{n}-\mathfrak{G}_{\text {in }} f_{n}$. Then $\varphi_{n} \rightarrow \kappa f^{0}$. Since $f_{n} \in$ $B(\mathbb{S}), G_{\text {in }} \varphi_{n}=f_{n}$. Passing to the limit, we obtain, by virtue of Fatou's Lemma, that $f^{0} \geq G_{\text {in }} \kappa f^{0}$, whence $f^{0} \in D\left(G_{\text {in }}\right)$.

Now we have $\varphi_{n}=(1+\mu) f_{n}-\mu \Pi f_{n}$. Since $\Pi f_{n} \leq \Pi f^{0} \leq c f^{0}$, we have $\left|\varphi_{\nu}(x)\right| \leq c_{1} f^{0}(x), x \in \mathbb{S}$, where $c_{1}>0$ is a constant. Thus, by the fact that $f^{0} \in$ $D\left(G_{\text {in }}\right)$, the functions $\varphi_{n}$ are bounded by an integrable in $g(x, d y)$ function $c_{1} f^{0}$. Therefore, $f^{0}=\lim _{n \rightarrow \infty} f_{n}=\lim _{n \rightarrow \infty} G_{\text {in }} \varphi_{n}=G_{\text {in }} \kappa f^{0}$. Hence, function $f^{0}$ satisfies Condition S2.

4.2 The analysis of the necessity conditions for BJM processes starts with the following intermediate result.

Theorem 4.2: Suppose that IM process $\xi$ is jump Markov and satisfies assumption (iii) of Section 2.2. Suppose that the corresponding BJM process $\Xi$ satisfies Condition N. Then $\Xi$ satisfies Condition NJ.

Proof: If function $\theta \in B(\mathbb{S})$, then

$$
\mathfrak{S}_{\text {in }} G_{\text {in }} \theta=G_{\text {in }} \mathfrak{F}_{\text {in }} \theta=G_{\text {in }} \theta-\theta .
$$

Consider an arbitrary nondecreasing sequence of functions $f_{n} \in B(\mathbb{S})$ converging to the function $f^{0}$ figuring in Condition N. As $\kappa f^{0} \geq f^{0}$, function $f^{0}$ is integrable in $\mathcal{G}(x, d y)$ for all $x \in \mathbb{S}$. Set $\varphi_{n}=G_{\text {in }} \kappa f_{n}$. As $f_{n} \uparrow f^{0}$, sequence $\varphi_{n}$ is nondecreasing in $n$. Also, $\varphi_{n} \leq G_{\mathrm{in}} \kappa f^{0} \leq f^{0}$. Thus, sequence $\varphi_{n}$ converges. Set $\varphi=\lim _{n \rightarrow \infty} \varphi_{n}$. Then $\varphi_{n} \leq \varphi \leq f^{0}$, and $\varphi$ is integrable in $\mathrm{G}(x, d y)$.

As $f_{n} \in B(\mathbb{S})$, we have, by virtue of (4.3) (with $\theta=\kappa f_{n}$ ), that $\varphi_{n} \in B(\mathbb{S})$ and

$$
\mathfrak{5} \varphi_{n}\left(=\mathfrak{F}_{\text {in }} \varphi_{n}\right)=\varphi_{n}-\kappa f_{n} \text {. }
$$

Thus, $-\mu \varphi_{n}+\mu \Pi \varphi_{n}=\varphi_{n}-\kappa f_{n}$, where $\Pi$ is defined in (4.2). Hence, $\mu \Pi \varphi_{n}=$ $(1+\mu) \varphi_{n}-\kappa f_{n}$, whence $\mu \Pi \varphi_{n} \leq c \varphi_{n}$, where $c>0$ is a constant. Furthermore, $\lim _{n \rightarrow \infty} \mu \Pi \varphi_{n}=(1+\mu) \varphi-\kappa f$. Since $\Pi$ is an integral operator, we obtain, by Fatou's 
Lemma, that $\lim _{n \rightarrow \infty} \mu \Pi \varphi_{n} \geq \mu \Pi \varphi$. Thus, $\varphi$ is integrable in $\pi(x, d y)$ for all $x \in \mathbb{S}$. Since $\varphi_{n} \leq \varphi$, by Lebesgue's Dominant Convergence Theorem, $\lim _{n \rightarrow \infty} \mathfrak{5} \varphi_{n}=\mathfrak{S}_{\text {in }} \varphi$. On the other hand, by (4.4), $\lim _{n \rightarrow \infty} \mathfrak{5} \varphi_{n}=\varphi-\kappa f$. Therefore, $\mathfrak{S}_{\text {in }} \varphi=\varphi-\kappa f \leq \varphi-\kappa \varphi$.

Thus, we have found a nonnegative function that satisfies the inequality in Condition NJ. It remains to check that $\varphi(x) \rightarrow \infty$ as $x \rightarrow \infty$. To this end, observe that $\mathfrak{5} \varphi_{n}(x)=-\mu(x) \varphi_{n}(x)+\mu(x) \Pi \varphi_{n}(x)$ (here, as before, $\varphi_{n}=G_{\text {in }} \kappa f_{n}$ ). Thus, $\left|\mathfrak{S} \varphi_{n}(x)\right| \leq c_{1} \varphi(x)$, where $c_{1}>0$ is a constant. We see that function $\left|\mathfrak{S} \varphi_{n}\right|$ is bounded by a function integrable in $\mathcal{G}(x, d y)$ for all $x \in \mathbb{S}$. Since $\varphi_{n} \in B(\mathbb{S}), G \mathfrak{S} \varphi_{n}=$ $G \varphi_{n}-\varphi_{n}$. Letting $n \rightarrow \infty$, we obtain $G_{\text {in }} \mathfrak{F}_{\text {in }} \varphi=G_{\text {in }} \varphi-\varphi$. On the other hand, as $\mathfrak{F}_{\text {in }} \varphi \leq \varphi-\kappa \varphi$, we have that $G_{\text {in }} \mathfrak{S}_{\text {in }} \varphi \leq G_{\text {in }} \varphi-G_{\text {in }} \kappa \varphi$. Therefore, $G_{\text {in }} \kappa \varphi \leq \varphi$. Relation $\lim _{x \rightarrow \infty} \varphi(x)=\infty$ now follows from Remark 2.5.

4.3 In this section, we establish the necessity of Condition $\mathrm{N}$ for quasiboundedness of a BJM process on a denumerable phase space $\mathbb{S}$, under assumption (iii) of Section 2.2 and the following additional assumption: (iv) there exists a point $x_{0}$ such that any point $x \in \mathbb{S}$ is accessible from $x_{0}$ by a finite number of jumps with transition probability $\pi$. That is, there exists a finite sequence $x_{0}, x_{1}, \ldots, x_{s}=x$ of points from $\mathbb{S}$ such that $\left.\pi\left(x_{i} x_{i+1}\right\}\right)>0, i=0, \ldots, s-1$.

Theorem 4.3: Suppose that for a BJM process $\Xi$, with an IM process $\xi$ on a denumerable set $\mathbb{S}$, assumptions (iii) and (iv) hold. Then if $\Xi$ is quasibounded, Condition $\mathrm{N}$ holds true.

A direct corollary of Theorems 4.2 and 4.3 is the following theorem.

Theorem 4.4: Under the conditions of Theorem 4.3, if $\Xi$ is quasibounded then Condition NJ holds true.

Proof of Theorem 4.3: For any bounded function $\varphi: S \mapsto \mathbb{R}$,

$$
\int \mathfrak{G}(x, d y) \varphi(y)=\sum_{y \in \mathbb{S}} q(x, y) \varphi(y) .
$$

Here $q(x, y)=\int \mathcal{G}(x, d u) I_{y}(u)$ and $I_{y}$ is the indicator of the one-point set $\{y\}$. Condition $(i v)$ implies that

$$
q\left(x_{0}, y\right)>0, y \in \mathbb{S} \text {. }
$$

According to (2.11) and (4.5),

It is easy to see that $\tilde{\psi}(y, v) \geq v$, whence

$$
\tilde{F}^{c}(x, z)=\sum_{y \in \mathbb{S}} q(x, y) \tilde{\psi}\left(y, \tilde{F}^{c}(y, z)\right) .
$$

$$
\widetilde{F}^{c}(x, y) \geq \sum_{y \in \mathbb{S}} q(x, y) \tilde{F}^{c}(y, z) .
$$

We again set

$$
\frac{\widetilde{F}^{c}(x, z)}{\widetilde{F}^{c}\left(x_{0}, z\right)}=H(x, z) \text {. }
$$

Then from (4.7) (with $x=x_{0}$ ) and (4.6) we get

$$
H(y, z) \leq \frac{1}{q\left(x_{0}, y\right)}, y \in \mathbb{S} .
$$


Thus, there exists a sequence of points $z_{n} \rightarrow \infty$ from $\mathbb{S}$ such that for any $y \in \mathbb{S}$ there exists the limit $\lim _{n \rightarrow \infty} H\left(y, z_{n}\right)$. Taking $f^{0}(y)$ equal to this limit leads, as in Section 2.4 , to the conclusion of Theorem 4.3.

\section{Acknowledgements}

F.I. Karpelevich thanks the American Mathematical Society and the International Science Foundation for support towards this work, and St. John's College, Cambridge, for warm hospitality during Michaelmas Term, 1995. Yu.M. Suhov thanks the Institute for Mathematics and its Applications, University of Minnesota for hospitality during visits in 1993 and 1994, and the Isaac Newton Institute for Mathematical Sciences, University of Cambridge, for hospitality during the 1995 programs. Both authors thank INTAS for support towards this work. Special thanks go to Mrs. S. Shea-Simonds for correcting the style of the paper.

\section{References}

[1] Athreya, K. and Ney, P., Branching Processes, Springer, Berlin 1972.

[2] Biggins, J.D., The first and last birth problems for a multitype age-dependent branching process, Adv. Appl. Prob. 8 (1976), 446-459.

[3] Biggins, J.D., How fast does a general branching random walk spread?, In: Classical and Modern Branching Processes, (ed. by K.B. Athreya and P. Jagers), IMA Volumes in Mathematics and its Applications 84, Springer, New York (1996), 19-40.

[4] Birkhoff, G. and Rota, G.-C., Ordinary Differential Equations, Blaisdell Publishing Co., Waltham, MA 1969.

[5] Dynkin, E.B., Markov Processes Vol. I, Springer, Berlin 1964.

[6] Dynkin, E.B., Superprocesses and partial differential equations, Ann. Prob. 21 (1993), 1185-1262.

[7] Dynkin, E.B., Kuznetsov, S.E. and Skorokhod, A.V., Branching measure-valued processes, Prob. Theory Related Fields 99 (1994), 55-96.

[8] Feller, W., Generalized second order differential operators and their lateral conditions, Illinois J. Math. 1 (1957), 459-504.

[9] Hammersley, J.M., Postulates for subadditive processes, Ann. Prob. 2 (1974), 652-680.

[10] Hartman, P., Ordinary Differential Equations, Wiley, New York 1964.

[11] Itô, K. and McKean, H.P., Diffusion Processes and Their Sample Paths, Springer, Berlin 1965.

[12] Karpelevich, F.I., Kelbert, M.Ya. and Suhov, Yu.M., The branching diffusion, stochastic equations and traveling wave solutions to the equations of Kolmogorov-Petrovskii-Piskunov, In: Cellular Automata and Cooperative Systems (ed. by N. Boccara, E. Goles, S. Martinez and P. Picco), Kluwer, Dordrecht (1993), 343-366.

[13] Karpelevich, F.I., Kelbert, M.Ya. and Suhov, Yu.M., The boundedness of branching Markov processes, In: The Dynkin Festschrift. Markov Processes and Their Applications (ed. by M.I. Freidlin). Progress in Probability 34, Birk- 
häuser, Boston (1994), 143-153.

[14] Karpelevich, F.I. and Suhov, Yu.M., A criterion of boundedness of discrete branching random walk, In: Classical and Modern Branching Processes (ed. by K.B. Athreya and P. Jagers), IMA Volumes in Mathematics and its Applications 84, Springer, New York (1996), 141-156.

[15] Kingman, J.F.C., The first birth problem for an age-dependent branching process, Ann. Prob. 3 (1975), 790-801.

[16] Lalley, S. and Sellke, T., Limit theorems for the frontier of a one-dimensional branching diffusion, Ann. Prob. 20 (1992), 1310-1340.

[17] Lalley, S. and Sellke, T., Hyperbolic branching Brownian motion, Prob. Theory Related Field 108 (1997), 171-192.

[18] McKean, H.P., Application of Brownian motion to the equation of KolmogorovPetrovskii-Piskunov, Comm. Pure Appl. Math. 28 (1975), 323-331.

[19] Taira, K., Diffusion Processes and Partial Differential Equations, Academic Press, Boston 1986.

[20] Yosida, K., Functional Analysis, Springer, Berlin 1978. 


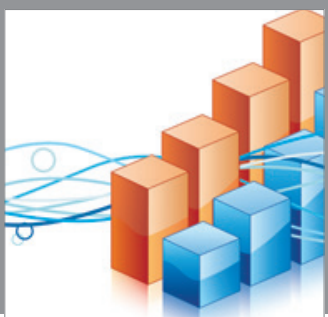

Advances in

Operations Research

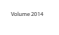

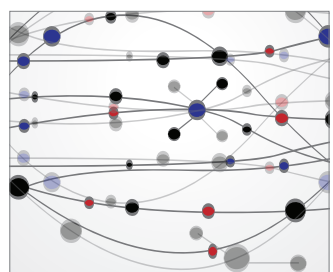

\section{The Scientific} World Journal
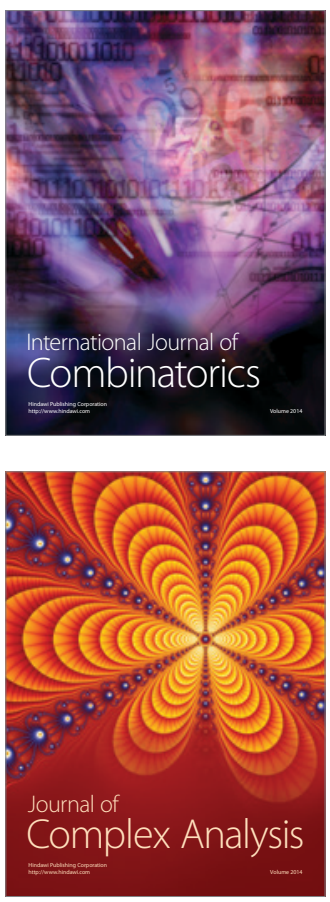

International Journal of

Mathematics and

Mathematical

Sciences
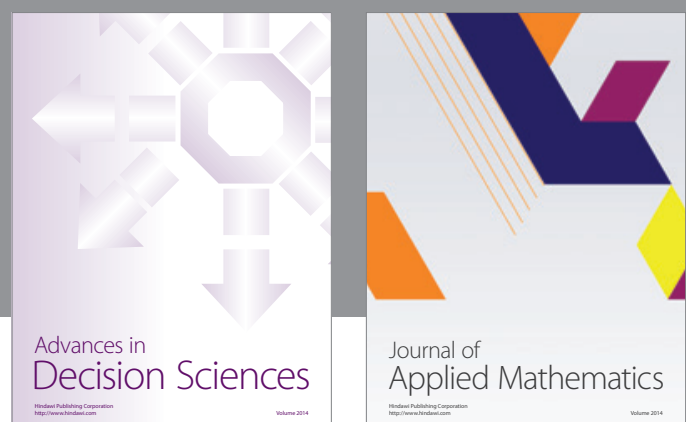

Journal of

Applied Mathematics
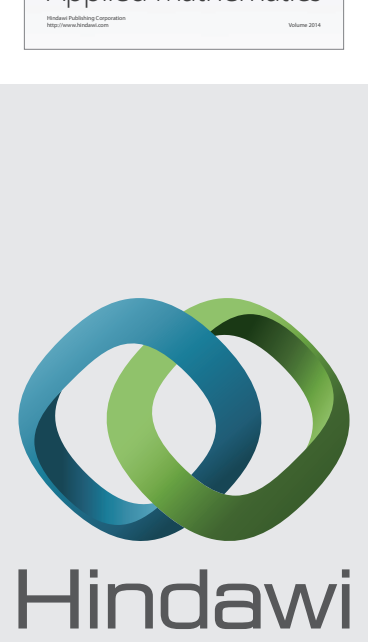

Submit your manuscripts at http://www.hindawi.com
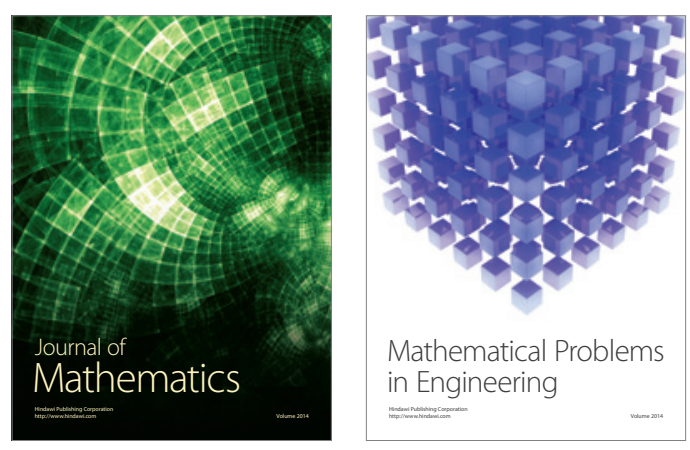

Mathematical Problems in Engineering
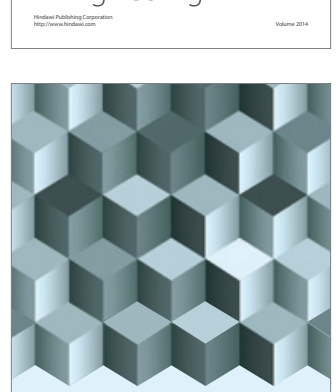

Journal of

Function Spaces
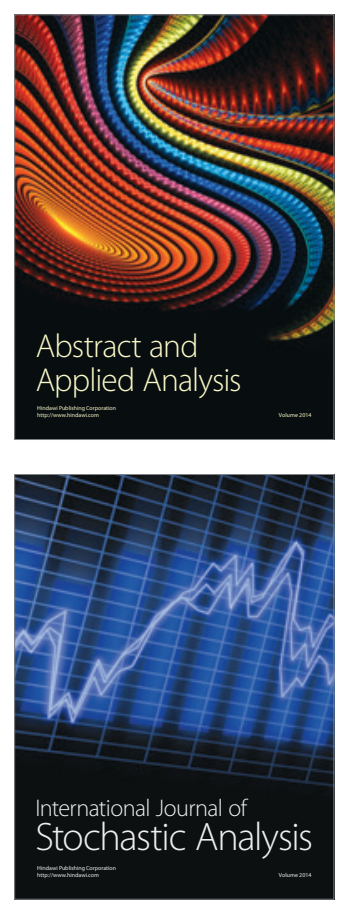

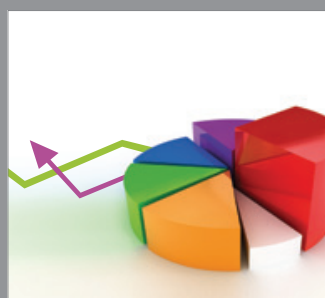

ournal of

Probability and Statistics

Promensencen
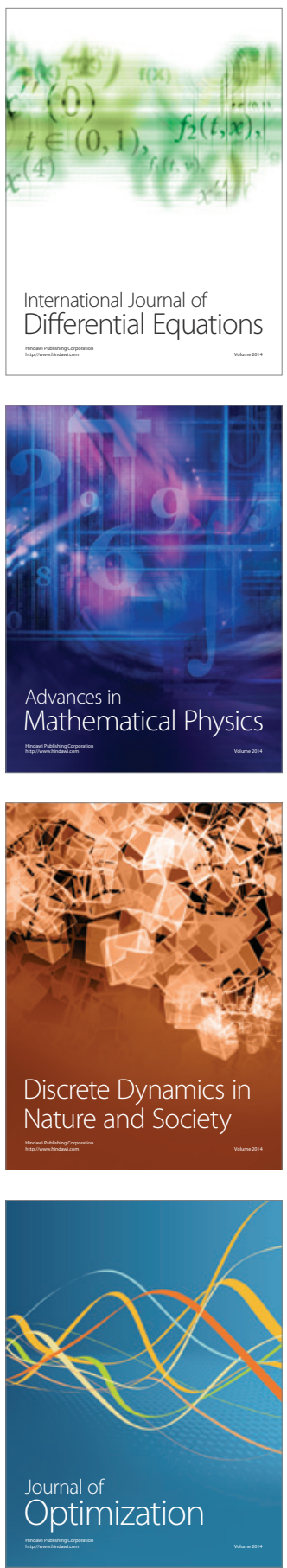